\title{
Stability of Frustration-Free Hamiltonians
}

\author{
Spyridon Michalakis \\ Institute for Quantum Information, Caltech, Pasadena, CA 9112蛙 \\ Justyna Pytel \\ Department of Physics, Nicolaus Copernicus University, Torun, Poland and \\ Department of Physics, Oregon State University, Corvallis, OR 97331
}

(Dated: October 4, 2011)

\begin{abstract}
We prove stability of the spectral gap for gapped, frustration-free Hamiltonians under general, quasi-local perturbations. We present a necessary and sufficient condition for stability, which we call Local Topological Quantum Order and show that this condition implies an area law for the entanglement entropy of the groundstate subspace. This result extends previous work by Bravyi et al. on the stability of topological quantum order for Hamiltonians composed of commuting projections with a common zero-energy subspace. We conclude with a list of open problems relevant to spectral gaps and topological quantum order.
\end{abstract}

\section{INTRODUCTION}

Recent interest in topological quantum computation has focused the attention of the condensed matter and mathematical physics community on Hamiltonians whose low-energy sectors exhibit some form of topological order. In a seminal paper by Kitaev [25], a Hamiltonian known as the toric code, was constructed out of commuting spininteraction terms, such that the groundstate subspace exhibits four-fold degeneracy on a torus, which may only be detected under macroscopic operations. In particular, the different groundstates are indistinguishable on microscopic and even macroscopic scales, which implies that local errors cannot create logical errors in any encoding that utilizes such topologically ordered groundstates as the encoded state of two qubits.

Nevertheless, if under a local perturbation the groundstate subspace were to split appreciably, or become mixed with the non-topological higher-energy sectors, then any guarantee of protection from errors would no longer be valid. At this point, another type of stability against errors is required, this time at the level of the spacing between the low-energy and high-energy sectors and in the form of a negligible splitting in the groundstate subspace. Progress in this direction was made by Klich [26] using the method of cluster expansions, and independently by Bravyi et al. 6. 8. In particular, the latter result showed that all Hamiltonians with commuting spin-interaction terms have negligible splitting in the groundstate subspace and a spectral gap that is stable under local perturbations, as long as the groundstates satisfy some type of local indistinguishability and frustration-freeness condition. Since the toric code Hamiltonian satisfies both conditions for gap stability, it follows that encoded qubits in the toric code groundstate subspace are robust against local errors, even if the Hamiltonian interactions are not precisely engineered. Moreover, at low enough temperatures, the spectral gap implies that the creation of excitations is unlikely to happen in relatively small time scales.

Even so, the toric code has a seemingly fatal flaw: at non-zero temperature, local excitations, once created, can travel around the torus to create logical errors at no extra energy cost. Motivated by this issue, soon after the results of Klich and Bravyi et al., a series of papers appeared that focused on the beneficial effects of Anderson-type localization of the undesired excitations in the presence of impurities, or external magnetic fields [24, 43, 45].

Still, until recently, no Hamiltonian model was known that combined the desirable properties of the toric code, with a rigorous and sufficiently large lower bound on the energy barrier restricting the mobility of unwanted excitations at non-zero temperature. In fact, it was shown by Yoshida [4], that such Hamiltonians would need to either forgo commutativity or break certain "natural" conditions, such as translational invariance or scale invariance, in order to satisfy stability at non-zero temperature. Indeed, the only known family of Hamiltonians with all the desired properties, recently presented by Haah in [14, has logical operators with fractal geometry in three dimensions and no scale invariance, thus sidestepping Yoshida's no-go theorem. Yet, despite the rigorous bound [5] on the energy barrier of Haah's Hamiltonian, there remains a question of whether the barrier is large enough to allow for operations on the logical qubits, like read-out and error-correction, which may require times comparable to the time it takes for logical

\footnotetext{
* spiros@caltech.edu

$\dagger$ letypka@fizyka.umk.pl
} 
errors to appear. Moreover, it was recently shown by Hastings [18, that all two-dimensional Hamiltonians which are a sum of commuting terms, have no topological order at non-zero temperature.

Motivated by this line of research and the larger question of the classification of quantum phases [3, 9, 39, 41, we present here a generalization of the result by Bravyi et al., which removes the commutativity of the Hamiltonian terms as an assumption for stability. Some of the new candidate Hamiltonians now include, parent Hamiltonians of Matrix Product States (MPS) and Projected Entangled Pair States (PEPS) [13, 36, 40, 41, as well as all other frustration-free Hamiltonians - that is, Hamiltonians whose groundstates minimize the energy of each local interaction term. Moreover, we generalize the conditions needed for the stability of the spectral gap, in hopes that in the future, one may be able to prove an equivalent result for general, gapped Hamiltonians, whose groundstates satisfy some type of topological order.

The stability of quantum phases was already studied by Borgs et al. [4] and Datta et al. [10, 11], where "classical" systems were shown to be robust against small quantum perturbations up to some low-temperature, using the methods of contour and cluster expansions. Nevertheless, here, we draw heavily from the methods developed in [6, 8], following the more succinct format of [6], which uses Hastings' powerful quasi-adiabatic continuation [21]. We make extensive use of Lieb-Robinson bounds [27, both for the evolution of operators according to the Hamiltonians under consideration [15, 19, 29, 33, 37, 38, as well as Lieb-Robinson bounds on the quasi-adiabatic evolution, or spectral flow, of gapped eigenspaces [3, 17, 20].

The paper is organized as follows: In Sec. II], we define the class of Hamiltonians whose stability we proceed to study. In Sec. III we introduce the conditions sufficient for proving stability, clarifying with examples the extend to which the conditions are also necessary. In Sec. IV] we define the class of perturbations we allow and proceed to transform the perturbed Hamiltonian, through a unitary process and a global energy shift, in a form amenable to studying its low energy sectors. Sec. V contains the proof of the bound on the energy of the perturbation with respect to arbitrary states, relative to the energy of the unperturbed Hamiltonian. The main theorem appears in Sec. VI and in Sec. VII we close with a discussion of future work and a list of open problems. Finally, Appendix A contains the technical details behind the estimate in Lemma 2 .

\section{THE HAMILTONIAN}

We study the stability of the spectral gap for Hamiltonians $H_{0}$ defined on $\Lambda=[0, L]^{d} \subset \mathbb{Z}^{d}$, satisfying:

1. (Spatially-local) $H_{0}=\sum_{u \in \Lambda} Q_{u}$, where each interaction $Q_{u}$ is a projection acting non-trivially on $b_{u}(1)$, where $b_{u}(r)$ is the ball of radius $r$ with center at site $u \in \Lambda$.

2. (Periodic-boundary) The set $\Lambda$ satisfies periodic boundary conditions.

3. (Frustration-free) For $P_{0}$ the projector onto the groundstate subspace of $H_{0}$, we have $H_{0} P_{0}=0$.

4. (Gapped) $H_{0}$ has a spectral gap $\gamma_{L} \geq \gamma>0$, for all $L \geq 2$.

Recall that $L$ is the linear size of the support of $H_{0}$, so when we write $H_{0}$, we assume a fixed size $L$. The gap condition implies that as $H_{0}$ is defined (translationally invariant, or otherwise) for larger $L$, the gap $\gamma_{L}$ remains uniformly bounded from below. The restriction of each interaction on a ball of radius 1 is not crucial, due to coarsegraining, as long as the interactions have constant range of support. Moreover, the assumption that the interactions are projections is not crucial either, as long as $Q_{u} P_{0}=\lambda_{u} P_{0}$, where $\lambda_{u}$ is the minimum eigenvalue of $Q_{u}$. This condition is the most general way of defining a frustration-free Hamiltonian. From now on, however, we will assume that $\lambda_{u}=0$, which follows from substituting $Q_{u}$ with $Q_{u}-\lambda_{u} \mathbb{1}$. Notice that this transformation corresponds to a global energy shift by $-\sum_{u} \lambda_{u} \mathbb{1}$, which leaves the spectral gap, and all other relevant properties of the Hamiltonian, unchanged.

\section{ASSUMPTIONS FOR STABILITY}

We begin this section by defining the following projections onto low-energy eigenstates:

Definition 1. For $B=b_{u}(r), r \geq 2$, we define $P_{B}(\epsilon)$ to be the projection onto the subspace of eigenstates of $H_{B}:=\sum_{b_{v}(1) \subset B} Q_{v}$ with energy at most $\epsilon \geq 0$. We set $P_{B}:=P_{B}(0)$.

The above definition implies the following relations, which hold for all frustration-free systems:

Corollary 1. The following claims follow from the definition of $P_{B}(\epsilon)$ : 
- $H_{B} \geq \epsilon\left(1-P_{B}(\epsilon)\right)$.

- $P_{B}(\epsilon) P_{B}\left(\epsilon^{\prime}\right)=P_{B}\left(\epsilon^{\prime}\right)$, for $0 \leq \epsilon^{\prime} \leq \epsilon$ and $P_{\Lambda}(\epsilon)=P_{0}$, for $0 \leq \epsilon \leq \gamma$.

- $P_{B}(\epsilon) P_{C}(0)=P_{C}(0)$, for $B \subset C$ and $\epsilon \geq 0$.

- $P_{B}(\epsilon)=P_{B}(0)$ if $H_{B}$ has spectral gap greater than $\epsilon$.

Proof. The first claim follows directly from the definition of $P_{B}(\epsilon)$, since $1-P_{B}(\epsilon)$ includes only states with energy at least $\epsilon$ in $H_{B}$. The second claim follows from the fact that $1-P_{B}(\epsilon)$ is orthogonal to $P_{B}\left(\epsilon^{\prime}\right)$ for $\epsilon^{\prime} \leq \epsilon$. Moreover, $P_{\Lambda}(\epsilon)=P_{\Lambda}\left(\epsilon^{\prime}\right)=P_{0}$, for all $\epsilon^{\prime} \in[0, \epsilon]$, since $H_{0}$ has spectral gap $\gamma \geq \epsilon$. The third claim follows from the second claim and $P_{B}(0) P_{C}(0)=P_{C}(0)$, which is true for frustration-free Hamiltonians since $H_{C} P_{C}(0)=0$ implies $H_{B} P_{C}(0)=0$. In particular, we get: $P_{B}(\epsilon) P_{C}(0)=\left(P_{B}(\epsilon) P_{B}(0)\right) P_{C}(0)=P_{C}(0)$. The fourth claim follows from the definition of $P_{B}(\epsilon)$ and the spectral decomposition of $H_{B}$.

We are now ready to introduce the main assumption needed for the stability of frustration-free systems. It is a condition on the degree of topological order for local ground states, so we shall call it Local Topological Quantum Order.

\section{A. Topological Order Assumption.}

Assumption 1. [Local-TQO] Let $A=b_{u}(r)$ with $r \leq L^{*}<L$ and $O_{A}$ be any bounded operator supported on A. Set $A(\ell)=b_{u}(r+\ell)$ and define

$$
c_{\ell}\left(O_{A}\right):=\operatorname{Tr}\left(P_{A(\ell)} O_{A}\right) / \operatorname{Tr} P_{A(\ell)}, \quad c\left(O_{A}\right):=\operatorname{Tr}\left(P_{0} O_{A}\right) / \operatorname{Tr} P_{0} .
$$

Then, for each fixed $1 \leq \ell \leq L-r$, we have:

$$
\left\|P_{A(\ell)} O_{A} P_{A(\ell)}-c_{\ell}\left(O_{A}\right) P_{A(\ell)}\right\| \leq\left\|O_{A}\right\| \Delta_{0}(\ell),
$$

where $\Delta_{0}(\ell)$ is a decaying function of $\ell$.

The cut-off parameter for topological order, $L^{*}$, may be thought of as the code distance of the encoding one may define on the (degenerate) groundstate space of $H_{0}$. Since we are interested in the stability of the spectral gap of $H_{0}$, we expect that $L^{*}$ should scale with $L$. To see this, note that if the Local-TQO condition fails after some fixed distance, then there must be operators $O_{A}$, with $A$ of increasing diameter and $\left\|O_{A}\right\|=1$, such that $\left\langle\Psi_{0}^{1}, O_{A} \Psi_{0}^{1}\right\rangle-\left\langle\Psi_{0}^{2}, O_{A} \Psi_{0}^{2}\right\rangle \geq c_{0}$, for some constant $c_{0}>0$ and $\left\{\left|\Psi_{0}^{1}\right\rangle,\left|\Psi_{0}^{2}\right\rangle\right\}$ groundstates of $H_{0}$. If we assume that the number of these operators $O_{A}$, for a given size of their support $A$, scales with $L$, then it follows that perturbing $H_{0}$ by $\sum_{A} O_{A}$, would create a macroscopic energy gap within the groundstate subspace, thus destroying any notion of stability and macroscopic order. This is exactly what happens in the case of the two-dimensional Ising Hamiltonian $H_{0}=-\sum_{p \sim q} \sigma_{p}^{z} \sigma_{q}^{z}$, under a uniform magnetic field $J \sum_{p} \sigma_{p}^{z}$. In particular, the two-fold degenerate groundstate space fails to satisfy Local-TQO at the most basic level, since any local operator $\sigma_{p}^{z}, p \in \mathbb{Z}^{2}$ can distinguish the groundstates (one has eigenvalue +1 and the other -1 .)

One may wonder at this point why it is necessary to demand that low-energy states are locally indistinguishable at all scales, and not just at the global scale of the system. In other words, why do we care if the groundstates of the local Hamiltonians are also topologically ordered? The reason is best exemplified with the following counterexample: Let $H_{0}=-\sigma_{0}^{z}-\sum_{p \sim q} \sigma_{p}^{z} \sigma_{q}^{z}$, where $p, q \in \mathbb{Z}^{2}$. This is a gapped Hamiltonian with a unique groundstate $|0 \cdots 0\rangle$, satisfying topological order at the global level. It is easy to see that a perturbation of the form $J \sum_{p \in \Lambda} \sigma_{p}^{z}$, will close the gap between the states $|000 \cdots 0\rangle$ and $|111 \cdots 1\rangle$, even for $J \sim 1 /|\Lambda|$. Since Local-TQO fails for any region that does not include the origin, this implies that some form of Local-TQO is necessary to protect the spectral gap from collapsing. The above examples validate our intuition that Hamiltonians are unstable because local "order parameters" can act as perturbations to either open the gap between groundstates, or close the gap between global groundstates and excited states which have low energy locally. In that sense, the above condition implies that states that minimize the energy of the Hamiltonian as more and more interactions are added, differ only near the boundary of the region we are considering.

The Local-TQO condition defined above, combines and generalizes assumptions $T Q O-1,2$ of the stability result for the commuting case [6, 8]. It is worth noting that the above condition is a property of the local, low-energy subspaces. In fact, it is possible in many cases (e.g. Hamiltonians with a unique Matrix Product State as their groundstate, or injective PEPS [36, [40, 41]) to modify a frustration-free Hamiltonian $H_{0}$ to a different Hamiltonian $H_{1}$, satisfying 
Local-TQO without changing the global ground state subspace, or closing the gap. In such cases, $H_{0}$ and $H_{1}$ are both gapped, frustration-free and share the same ground state space, so $H_{s}=(1-s) H_{0}+s H_{1}$ is also gapped and frustration-free with the same ground state space. In other words, if $H_{1}$ satisfies Local-TQO and $H_{0}$ does not, we can apply the stability result to $H_{1}$ to infer properties about the groundstate of $H_{0}$. For a concrete example, one can look at two different toric code Hamiltonians, as discussed in [8, Sec. 2.4].

Corollary 2. Let $O_{A}$ be any bounded operator supported on the ball $A=b_{u}(r)$, with $r \leq L^{*}$ and $u \in \Lambda$. Then, Local-TQO implies the following bounds for $1 \leq \ell \leq L-r$ :

$$
\begin{aligned}
\left\|O_{A} P_{A(\ell)}\right\|-\left\|O_{A} P_{0}\right\| & \leq 2\left\|O_{A}\right\| \sqrt{\Delta_{0}(\ell)} . \\
\left\|P_{A(\ell)}-P_{A(\ell)} P_{A}\right\| & \leq \sqrt{3} \sqrt{\Delta_{0}(\ell)} .
\end{aligned}
$$

Proof. From Local-TQO and the triangle inequality, we have:

$$
\begin{aligned}
\left|\left\|O_{A} P_{A(\ell)}\right\|^{2}-c_{\ell}\left(\left|O_{A}\right|^{2}\right)\right| & \leq\left\|O_{A}\right\|^{2} \Delta_{0}(\ell), \\
\left|\left\|O_{A} P_{0}\right\|^{2}-c_{L-r}\left(\left|O_{A}\right|^{2}\right)\right| & \leq\left\|O_{A}\right\|^{2} \Delta_{0}(L-r), \\
\left|c_{L-r}\left(\left|O_{A}\right|^{2}\right)-c_{\ell}\left(\left|O_{A}\right|^{2}\right)\right| & \leq\left\|O_{A}\right\|^{2}\left(\Delta_{0}(\ell)+\Delta_{0}(L-r)\right) .
\end{aligned}
$$

The last inequality follows from a triangle inequality on (4) and the bound:

$$
\left\|P_{0}\left|O_{A}\right|^{2} P_{0}-c_{\ell}\left(\left|O_{A}\right|^{2}\right) P_{0}\right\|=\left\|P_{0} P_{A(\ell)}\left[\left|O_{A}\right|^{2}-c_{\ell}\left(\left|O_{A}\right|^{2}\right)\right] P_{A(\ell)} P_{0}\right\| \leq\left\|P_{A(\ell)}\left|O_{A}\right|^{2} P_{A(\ell)}-c_{\ell}\left(\left|O_{A}\right|^{2}\right) P_{A(\ell)}\right\| .
$$

Recalling that $\Delta_{0}(\ell)$ is decaying, we have:

$$
\|\| O_{A} P_{A(\ell)}\|-\| O_{A} P_{0}\left\|\left.\right|^{2} \leq\left|\left\|O_{A} P_{A(\ell)}\right\|^{2}-\left\|O_{A} P_{0}\right\|^{2}\right| \leq 2\right\| O_{A}\left\|^{2}\left(\Delta_{0}(\ell)+\Delta_{0}(L-r)\right) \leq 4\right\| O_{A} \|^{2} \Delta_{0}(\ell) .
$$

The second bound is equivalent to showing that $\left\|\left(1-P_{A}\right) P_{A(\ell)}\right\|^{2} \leq 3 \Delta_{0}(\ell)$. If we are given two projections $P$ and $Q$ satisfying: $\|P Q P-P\| \leq \delta$, then we have $\|P(1-Q) P\| \leq \delta \Longrightarrow\|(1-Q) P\|^{2} \leq \delta$. In our case, we have from LocalTQO: $\left\|P_{A(\ell)} P_{A} P_{A(\ell)}-P_{A(\ell)}\right\| \leq 2 \Delta_{0}(\ell)+\Delta_{0}(L-r)$, where we used Corollary 1 to get $c_{L-r}\left(P_{A}\right)=\operatorname{Tr}\left(P_{A} P_{0}\right) / \operatorname{Tr} P_{0}=1$ and combined it with (5) and a triangle inequality.

Note that in the above Corollary, the projections $P_{A}$ and $P_{A(\ell)}$ correspond to $P_{A}(\epsilon)$ and $P_{A(\ell)}(\epsilon)$, respectively. All that is needed for the bounds to hold is that the projections satisfy the Local-TQO condition. Of course, for $P_{A}=P_{A}(0)$ and $P_{A(\ell)}=P_{A(\ell)}(0)$, we have from Corollary 1 that $\left\|P_{A(\ell)}-P_{A(\ell)} P_{A}\right\|=0$.

The next Corollary makes precise the notion of local indistinguishability between ground states and provides an equivalent condition to Local-TQO.

Corollary 3. Let $A=b_{u}(r)$, with $r \leq L^{*}$ and $u \in \Lambda$. For any two groundstates $\left|\psi_{1}(A(\ell))\right\rangle,\left|\psi_{2}(A(\ell))\right\rangle$ of $H_{A(\ell)} ;$ that is, $P_{A(\ell)}\left|\psi_{i}(A(\ell))\right\rangle=\left|\psi_{i}(A(\ell))\right\rangle, i=1,2$, define the reduced density matrices $\rho_{i}(A)=\operatorname{Tr}_{A(\ell) \backslash A}\left|\psi_{i}(A(\ell))\right\rangle\left\langle\psi_{i}(A(\ell))\right|$. Then, Local-TQO is equivalent to:

$$
\left\|\rho_{1}(A)-\rho_{2}(A)\right\|_{1} \leq 2 \Delta_{0}(\ell)
$$

Proof. First, we prove that Local-TQO implies the above condition. Set $\rho_{i}=\left|\psi_{i}(A(\ell))\right\rangle\left\langle\psi_{i}(A(\ell))\right|, i=1,2$. Then, $\rho_{i}(A)=\operatorname{Tr}_{A(\ell) \backslash A} \rho_{i}$ and is supported only on $A$. From Local-TQO, for any operator $O_{A}$ supported on $A$ :

$$
\left|\operatorname{Tr}\left(\rho_{1}-\rho_{2}\right) O_{A}\right|=\left|\left\langle\psi_{1}(A(\ell))\left|O_{A}-c_{\ell}\left(O_{A}\right)\right| \psi_{1}(A(\ell))\right\rangle-\left\langle\psi_{2}(A(\ell))\left|O_{A}-c_{\ell}\left(O_{A}\right)\right| \psi_{2}(A(\ell))\right\rangle\right| \leq 2\left\|O_{A}\right\| \Delta_{0}(\ell) .
$$

Now, recalling that $\left\|\rho_{1}(A)-\rho_{2}(A)\right\|_{1}=\sup _{O_{A}:\left\|O_{A}\right\|=1}\left|\operatorname{Tr}\left(\rho_{1}-\rho_{2}\right) O_{A}\right|$, we get the desired bound. To prove the other direction, we note that it is sufficient to show that for $\left|\Psi_{0}(A(\ell))\right\rangle$ satisfying $P_{A(\ell)}\left|\Psi_{0}(A(\ell))\right\rangle=\left|\Psi_{0}(A(\ell))\right\rangle$, we have:

$$
\left|\left\langle\Psi_{0}(A(\ell))\left|O_{A}\right| \Psi_{0}(A(\ell))\right\rangle-c_{\ell}\left(O_{A}\right)\right| \leq 2\left\|O_{A}\right\| \Delta_{0}(\ell)
$$

Setting $N=\operatorname{Tr} P_{A(\ell)}-1$ and $\rho_{k}(A)=\operatorname{Tr}_{A(\ell) \backslash A}\left|\Psi_{k}(A(\ell))\right\rangle\left\langle\Psi_{k}(A(\ell))\right|, \quad 0 \leq k \leq N$, where $\left\{\left|\Psi_{k}(A(\ell))\right\rangle\left\langle\Psi_{k}(A(\ell))\right|\right\}_{k=0}^{N}$ is an orthogonal decomposition for $P_{A(\ell)}$, we have for $\left\|O_{A}\right\|=1$ :

$$
\begin{aligned}
& \left|\left\langle\Psi_{0}(A(\ell))\left|O_{A}\right| \Psi_{0}(A(\ell))\right\rangle-c_{\ell}\left(O_{A}\right)\right|=\left|\operatorname{Tr} O_{A} \rho_{0}(A)-c_{\ell}\left(O_{A}\right)\right|= \\
& \frac{1}{N+1}\left|\sum_{k=0}^{N} \operatorname{Tr} O_{A}\left(\rho_{0}(A)-\rho_{k}(A)\right)\right| \leq \frac{1}{N+1} \sum_{k=1}^{N}\left\|\rho_{0}(A)-\rho_{k}(A)\right\|_{1} \leq \frac{N}{N+1} \Delta_{0}(\ell) .
\end{aligned}
$$

Hence, up to constant pre-factors, 6 and Local-TQO are equivalent. 
Moreover, Local-TQO implies an area law [16] for the entanglement entropy of the ground states of $H_{0}$.

Corollary 4. Let $A=b_{u}(r)$, with $r \leq L^{*}$ and $u \in \Lambda$. Any groundstate $\left|\Psi_{0}\right\rangle$ of $H_{0}$ satisfying Local-TQO, also satisfies an area law for the entanglement entropy of $\rho_{A}:=\operatorname{Tr}_{A^{c}}\left|\Psi_{0}\right\rangle\left\langle\Psi_{0}\right|$ :

$$
S\left(\rho_{A}\right) \leq\left(c_{d} \ln D\right)(1+r)^{d-1} \cdot \ell_{0},
$$

where $c_{d}$ is a constant depending only on the dimension $d$ of the lattice, $D$ is the maximum dimension of the on-site Hilbert spaces (e.g. $D=2$ for spin-1/2 particles) and $\ell_{0}=\min \left\{\ell: \Delta_{0}(\ell) \leq \ell /(1+r)\right\}$.

Proof. We begin by writing the Schmidt decomposition of $\left|\Psi_{0}\right\rangle$ over the partition of the lattice $A(\ell): \Lambda \backslash A(\ell)$, where $\ell$ will be chosen later:

$$
\left|\Psi_{0}\right\rangle=\sum_{k \geq 0} \sqrt{\sigma_{k}}\left|\Psi_{k}(A(\ell))\right\rangle \otimes\left|\Psi_{k}(\Lambda \backslash A(\ell))\right\rangle, \quad\left\langle\Psi_{k}(\Lambda \backslash A(\ell)), \Psi_{l}(\Lambda \backslash A(\ell))\right\rangle=\delta_{k, l}, \quad \sum_{k \geq 0} \sigma_{k}=1, \quad \sigma_{k}>0
$$

Note that for frustration-free Hamiltonians, the states $\left\{\left|\Psi_{k}(A(\ell))\right\rangle\right\}_{k \geq 0}$ in the Schmidt decomposition of $\left|\Psi_{0}\right\rangle$ are groundstates of the Hamiltonian $H_{A(\ell)}$. To see this, recall that $H_{0}=\sum_{u} Q_{u}, Q_{u} \geq 0$ and $H_{0}\left|\Psi_{0}\right\rangle=0$, which implies that $\left\langle\Psi_{0}\left|H_{A(\ell)}\right| \Psi_{0}\right\rangle=0=\sum_{k \geq 0} \sigma_{k}\left\langle\Psi_{k}(A(\ell))\left|H_{A(\ell)}\right| \Psi_{k}(A(\ell))\right\rangle$, hence $H_{A(\ell)}\left|\Psi_{k}(A(\ell))\right\rangle=0, k \geq 0$. Setting $\rho_{k}(A)=\operatorname{Tr}_{A(\ell) \backslash A}\left|\Psi_{k}(A(\ell))\right\rangle\left\langle\Psi_{k}(A(\ell))\right|$, we get from $(9)$ :

$$
\rho_{A}:=\operatorname{Tr}_{\Lambda \backslash A}\left|\Psi_{0}\right\rangle\left\langle\Psi_{0}\right|=\sum_{k \geq 0} \sigma_{k} \rho_{k}(A)=\rho_{0}(A)+\sum_{k \geq 0} \sigma_{k}\left(\rho_{k}(A)-\rho_{0}(A)\right) .
$$

Corollary 3 and $(10)$ give: $\left\|\rho_{A}-\rho_{0}(A)\right\|_{1} \leq 2 \Delta_{0}(\ell)$. Combined with the bound $S\left(\rho_{o}(A)\right) \leq(|A(\ell)|-|A|) \ln D \leq$ $c_{d}^{\prime}(1+r)^{d-1} \ell \ln D$, for $\ell \leq r$ and $c_{d}^{\prime}$ a constant depending only on $d$, we have:

$$
S\left(\rho_{A}\right) \leq S\left(\rho_{0}(A)\right)+\left|S\left(\rho_{A}\right)-S\left(\rho_{0}(A)\right)\right| \leq c_{d}^{\prime} \ln D(1+r)^{d-1} \ell+c_{d}^{\prime \prime} \ln D \Delta_{0}(\ell)(1+r)^{d}+H\left(\Delta_{0}(\ell)\right)
$$

where we used the Fannes-Audenaert inequality [2, 12] for the entropy:

$$
\left|S\left(\rho_{A}\right)-S\left(\rho_{0}(A)\right)\right| \leq\left(\left\|\rho_{A}-\rho_{0}(A)\right\|_{1} / 2\right) \ln D^{|A|}+H\left(\left\|\rho_{A}-\rho_{0}(A)\right\|_{1} / 2\right),
$$

with $H(p)=-p \ln p-(1-p) \ln (1-p), p \in[0,1]$. Setting $\ell_{0}=\min \left\{\ell: \Delta_{0}(\ell) \leq \ell /(1+r)\right\}$ and $c_{d}=\max \left\{c_{d}^{\prime}, c_{d}^{\prime \prime}\right\}+1$, we get the desired bound, noting that $H\left(\Delta_{0}\left(\ell_{0}\right)\right) \leq \ln 2$.

We note that for stabilizer Hamiltonians, like the toric code, the Local-TQO decay $\Delta_{0}(\ell)$ vanishes after a constant length $\ell_{0}$; in other words, $\Delta_{0}(\ell)=0, \ell \geq \ell_{0}$. This implies that for such Hamiltonians, the groundstates satisfy an area law exactly. On the other hand, if the decay $\Delta_{0}(\ell)$ is exponential in $\ell$, then there is a logarithmic divergence $\left(\ell_{0} \sim \ln (1+r)\right)$ in the area law, which is natural for gapless systems (the above proof made no use of a spectral gap.)

\section{B. Local Gap Assumption}

The second assumption imposes a condition on the spectral gaps of the local Hamiltonians $H_{b_{u}(r)}$.

Definition 2. [Local-Gap] We say that $H_{0}$ is locally gapped with gap $\gamma(r)>0$, iff for each $u \in \Lambda$ and $r \geq 0$, $P_{b_{u}(r)}(\gamma(r))=P_{b_{u}(r)}$. When $\gamma(r)$ decays at most polynomially in $r$, we say that $H_{0}$ satisfies the Local-Gap condition.

Below, we present an example of a gapped, translationally-invariant, frustration-free Hamiltonian with Local-TQO, that is unstable under vanishingly small perturbations:

Example 1. Let $H_{2 N}=\sum_{k=1}^{2 N} H_{k, k+1}$, where $H_{k, k+1}=1 /(3 N)|0\rangle\left\langle\left. 0\right|_{k} \otimes \mid 1\right\rangle\left\langle\left. 1\right|_{k+1}+\mid 1\right\rangle\left\langle\left. 1\right|_{k} \otimes \mid 1\right\rangle\left\langle\left. 1\right|_{k+1}+\mid 0\right\rangle\left\langle\left. 0\right|_{k} \otimes\right.$ $|0\rangle\left\langle\left. 0\right|_{k+1}\right.$, for $k$ even and $\left.H_{k, k+1}=1 /(3 N) \mid 1\right\rangle\left\langle\left. 1\right|_{k} \otimes \mid 0\right\rangle\left\langle\left. 0\right|_{k+1}+\mid 1\right\rangle\left\langle\left. 1\right|_{k} \otimes \mid 1\right\rangle\left\langle\left. 1\right|_{k+1}+\mid 0\right\rangle\left\langle\left. 0\right|_{k} \otimes \mid 0\right\rangle\left\langle\left. 0\right|_{k+1}\right.$, for $k$ odd. There is a unique groundstate $|0101 \ldots 01\rangle$, with energy 0 and spectral gap $2 / 3$ to the first excited state $|1010 \ldots 10\rangle$. Note that since all eigenstates of $H_{2 N}$ can be written as product states over $|0\rangle,|1\rangle$, any state with consecutive 0 's, or 1 's has energy at least 1. It should be obvious that this Hamiltonian satisfies frustration-freeness and Local-TQO (trivially, since the groundstate is unique and is a product state), but is unstable under the perturbation $V=-\sum_{k=1}^{2 N} V_{k}$, where $V_{k}=1 /(3 N)|0\rangle\left\langle\left. 0\right|_{k} \otimes \mid 1\right\rangle\left\langle\left. 1\right|_{k+1}\right.$, for $k$ even and $\left.V_{k}=1 /(3 N) \mid 1\right\rangle\left\langle\left. 1\right|_{k} \otimes \mid 0\right\rangle\left\langle\left. 0\right|_{k+1}\right.$, for $k$ odd. Interestingly, the Hamiltonian $H_{2 N}+V$ is frustration-free, has degenerate ground state space and satisfies the Local-Gap condition, but not the Local-TQO assumption, since there exist local operators, like $|0\rangle\langle 0|\otimes| 1\rangle\langle 1|$, that distinguish the two ground states $\{|010101 \ldots 01\rangle,|101010 \ldots 10\rangle\}$. In other words, the two assumptions for stability seem to be independent and dual to each other. Moreover, this example suggests that, in some further refined form, both conditions are necessary for stability. 
Such violations to the Local-Gap condition are not obvious for Hamiltonians that are sums of non-vanishing terms. In fact, to the authors' knowledge, it remains an open problem whether it is possible to construct examples of gapped, frustration-free Hamiltonians that are sums of local projections and do not satisfy the Local-Gap condition. Moreover, we expect that the Local-Gap condition can be merged with a stronger version of the Local-TQO condition, where the projections $P_{A(\ell)}$ are substituted with $P_{A(\ell)}(\epsilon)$, for some $\epsilon<\gamma$. Furthermore, we expect that using the powerful detectability lemma 1, one may be able to remove the Local-Gap assumption, when the Hamiltonian interactions can be rescaled to projections in a system-size independent way.

\section{TRANSFORMING THE PERTURBED HAMILTONIAN}

We begin this section by defining the general class of perturbations we will be studying. The only restriction is a certain notion of quasi-locality that we make precise in the following definition:

Definition 3. We define a perturbation $V$ to have strength $J$ and decay $f_{0}(r)$, iff we can write $V=\sum_{u \in \Lambda} \sum_{r \geq 0}^{L} V_{u}(r)$, such that $V_{u}(r)$ has support on $b_{u}(r)$ and satisfies $\left\|V_{u}(r)\right\| \leq J f_{0}(r)$, for some rapidly decaying function $f_{0}(r)$. We write that $V$ is a $\left(J, f_{0}\right)$-perturbation.

We have chosen to leave the decay rate of $f_{0}$ ill-defined, beyond saying that it is rapidly-decaying, in order to avoid constraining the results that follow unnecessarily. Of course, throughout this paper, we assume that $f_{0}$ decays fast enough to allow for the usual Lieb-Robinson bounds [19, 33, on evolutions based on $H_{0}+V$. This implies that fixed range and exponentially-decaying perturbations are well within the purview of this paper.

In order to relate the spectrum of the initial Hamiltonian $H_{0}$ to the spectrum of the final Hamiltonian $H_{0}+V$, we define the following one-parameter family of gapped Hamiltonians:

Definition 4. Let $H_{s}:=H_{0}+s V$, where $H_{0}$ satisfies the assumptions of the previous sections and $V$ is a $\left(J, f_{0}\right)$ perturbation. We assume that $H_{s}$ has spectral gap $\gamma_{s} \geq \gamma^{\prime}>0$ and groundstate projection $P_{0}(s)$, for $s \in\left[0, s^{\prime}\right]$ and $s^{\prime} \in[0,1]$ the maximum value of $s$, such that the gap condition is satisfied.

Note that $s^{\prime}$ may depend on $\gamma^{\prime}, J$ and $f_{0}$, as well as the linear size $L$, of the system. Moreover, $\gamma^{\prime}$ is fixed a priori to some value less than $\gamma$. Concretely, we may take $\gamma^{\prime}=\gamma / 2$, but this is not crucial. The estimates below will depend implicitly on our choice of $\gamma^{\prime}$.

The main goal of this section is to transform a $\left(J, f_{0}\right)$-perturbation $V$, into a $(J, w)$-perturbation $W$ with similar decay, such that: $W_{u}(r) P_{b_{u}(r)}=0$ and for some unitary $U$ we have, $U^{\dagger}\left(H_{0}+V\right) U=H_{0}+W+\Delta+E \mathbb{1}$, where $\|\Delta\| \rightarrow 0$ as $L \rightarrow \infty$ and $E=\operatorname{Tr}\left(U P_{0} U^{\dagger}\left(H_{0}+V\right)\right) / \operatorname{Tr} P_{0}$. Recall that $P_{b_{u}(r)}$ is the ground state projector of the local, frustration-free Hamiltonian $H_{b_{u}(r)}$.

\section{A. Making the global ground states commute quasi-locally.}

In this section, we re-derive a version of Lemma 7 in [6], which will be sufficient for our purposes. The content of this Lemma can be summarized as the decomposition of any gapped Hamiltonian into a sum of quasi-local interactions that commute with the groundstate subspace. Note that any local, frustration-free Hamiltonian satisfies this condition (with strict locality), but once we add a perturbation, the new terms may no longer commute with the groundstate subspace.

Lemma 1. Let $H_{s}$ be the family of gapped Hamiltonians given in Definition 4. Then, there exists a linear operator $\mathcal{F}_{\gamma^{\prime}, s}$, with the following properties:

(i) $\mathcal{F}_{\gamma^{\prime}, s}\left(H_{s}\right)=H_{s}$ and, hence, $\mathcal{F}_{\gamma^{\prime}, 0}\left(H_{0}\right)=H_{0}$,

(ii) $\left[\mathcal{F}_{\gamma^{\prime}, s}\left(O_{u}(r)\right), P_{0}(s)\right]=0$, for any operator $O_{u}(r)$ supported on $b_{u}(r), u \in \Lambda$,

(iii) $\left\|\mathcal{F}_{\gamma^{\prime}, s}\left(O_{u}(r)\right)\right\| \leq\left\|O_{u}(r)\right\|$,

(iv) $\mathcal{F}_{\gamma^{\prime}, s}\left(O_{u}(r)\right)=\sum_{r^{\prime} \geq 0} \mathcal{F}_{\gamma^{\prime}, s}\left(r^{\prime} ; O_{u}(r)\right)$, with $\mathcal{F}_{\gamma^{\prime}, s}\left(r^{\prime} ; O_{u}(r)\right)$ supported on $b_{u}\left(r+r^{\prime}\right)$ and $\left\|\mathcal{F}_{\gamma^{\prime}, s}\left(r^{\prime} ; O_{u}(r)\right)\right\| \leq$ $\left\|O_{u}(r)\right\| f_{0}^{(1)}\left(r^{\prime}\right)$, for a rapidly decaying function $f_{0}^{(1)}$.

(v) $\mathcal{F}_{\gamma^{\prime}, s}\left(Q_{u}\right)-\mathcal{F}_{\gamma^{\prime}, 0}\left(Q_{u}\right)=\sum_{r \geq 2} X_{u}^{(1)}(r)$, with $X_{u}^{(1)}(r)$ supported on $b_{u}(r)$ and $\left\|X_{u}^{(1)}(r)\right\| \leq s J\left\|Q_{u}\right\| f_{1}^{(1)}(r)$, for a rapidly decaying function $f_{1}^{(1)}$. 
Proof. Define the operator $\mathcal{F}_{\gamma^{\prime}, s}$ as follows:

$$
\mathcal{F}_{\gamma^{\prime}, s}\left(O_{u}(r)\right)=\int_{-\infty}^{\infty} d t w_{\gamma^{\prime}}(t) \tau_{t}^{H_{s}}\left(O_{u}(r)\right)
$$

where $\tau_{t}^{B}(A)=e^{i t B} A e^{-i t B}$ and $w_{\gamma^{\prime}}(t)$ is the filter function studied in [3, 17, with the following properties:

1. Compact Fourier Transform $\left[22\right.$ : $\hat{w}_{\gamma^{\prime}}(\omega)=0$, for $|\omega| \geq \gamma^{\prime}$ and $\hat{w}_{\gamma^{\prime}}(0)=1$.

2. Rapid decay: $0 \leq w_{\gamma^{\prime}}(t) \leq 2 e^{2} \gamma^{\prime}\left(\gamma^{\prime}|t|\right) \exp \left(-\frac{2}{7} \frac{\gamma^{\prime}|t|}{\ln ^{2}\left(\gamma^{\prime}|t|\right)}\right)$, for $\gamma^{\prime}|t| \geq e^{1 / \sqrt{2}}$ and $0 \leq w_{\gamma^{\prime}}(t) \leq \gamma^{\prime} / \pi$, for all $t \in \mathbb{R}$. Note that, we immediately get:

$$
\mathcal{F}_{\gamma^{\prime}, s}\left(H_{s}\right)=\int_{-\infty}^{\infty} d t w_{\gamma^{\prime}}(t) e^{i t H_{s}}\left(H_{s}\right) e^{-i t H_{s}}=\left(\int_{-\infty}^{\infty} d t w_{\gamma^{\prime}}(t)\right) H_{s}=H_{s}
$$

since $\int_{-\infty}^{\infty} d t w_{\gamma^{\prime}}(t)=\hat{w}_{\gamma^{\prime}}(0)=1$. Moreover, the new interactions commute with $P_{0}(s)$, since for any ground state $\left|\psi_{0}(s)\right\rangle$ of $H_{s}$ and any eigenstate $\left|\psi_{n}(s)\right\rangle$ orthogonal to $P_{0}(s)$ with eigenvalue $E_{n}(s)$, we have:

$$
\left\langle\psi_{n}(s)\left|\mathcal{F}_{\gamma^{\prime}, s}\left(O_{u}(r)\right)\right| \psi_{0}(s)\right\rangle=\hat{w}_{\gamma^{\prime}}\left(E_{n}(s)-E_{0}(s)\right)\left\langle\psi_{n}(s)\left|O_{u}(r)\right| \psi_{0}(s)\right\rangle=0,
$$

where $E_{n}(s)-E_{0}(s) \geq \gamma^{\prime}$, by assumption. This implies that:

$$
\left(\mathbb{1}-P_{0}(s)\right) \mathcal{F}_{\gamma^{\prime}, s}\left(O_{u}(r)\right) P_{0}(s)=0=P_{0}(s) \mathcal{F}_{\gamma^{\prime}, s}\left(O_{u}(r)\right)\left(1-P_{0}(s)\right),
$$

hence $\mathcal{F}_{\gamma^{\prime}, s}\left(O_{u}(r)\right) P_{0}(s)=P_{0}(s) \mathcal{F}_{\gamma^{\prime}, s}\left(O_{u}(r)\right) P_{0}(s)=P_{0}(s) \mathcal{F}_{\gamma^{\prime}, s}\left(O_{u}(r)\right)$, as desired. We also have by the triangle inequality:

$$
\left\|\mathcal{F}_{\gamma^{\prime}, s}\left(O_{u}(r)\right)\right\| \leq \int_{-\infty}^{\infty} d t w_{\gamma^{\prime}}(t)\left\|e^{i t H_{s}}\left(O_{u}(r)\right) e^{-i t H_{s}}\right\|=\left\|O_{u}(r)\right\| .
$$

Finally, the quasi-locality of $\mathcal{F}_{\gamma^{\prime}, s}\left(O_{u}(r)\right)$ follows from writing $\mathcal{F}_{\gamma^{\prime}, s}\left(O_{u}(r)\right)=\sum_{r^{\prime} \geq 0} \mathcal{F}_{\gamma^{\prime}, s}\left(r^{\prime} ; O_{u}(r)\right)$, where for $r^{\prime} \geq 1$ :

$$
\mathcal{F}_{\gamma^{\prime}, s}\left(r^{\prime} ; O_{u}(r)\right)=\int_{-\infty}^{\infty} d t w_{\gamma^{\prime}}(t)\left(\tau_{t}^{H_{s}^{u}\left(r+r^{\prime}\right)}\left(O_{u}(r)\right)-\tau_{t}^{H_{s}^{u}\left(r+r^{\prime}-1\right)}\left(O_{u}(r)\right)\right),
$$

and

$$
\mathcal{F}_{\gamma^{\prime}, s}\left(0 ; O_{u}(r)\right)=\int_{-\infty}^{\infty} d t w_{\gamma^{\prime}}(t) \tau_{t}^{H_{s}^{u}(r)}\left(O_{u}(r)\right)
$$

with the Hamiltonians in the exponent defined as follows, for $0 \leq q \leq L$ :

$$
H_{s}^{u}(q)=\sum_{v: b_{v}(1) \subset b_{u}(q)} Q_{v}+\sum_{(k, v): b_{v}(k) \subset b_{u}(q)} s \cdot V_{v}(k) .
$$

Note that $H_{s}^{u}(L)=H_{s}$ and that $\mathcal{F}_{\gamma^{\prime}, s}\left(r^{\prime} ; O_{u}(r)\right)$ has support on $b_{u}\left(r+r^{\prime}\right)$ and norm decaying rapidly in $r^{\prime}$. The decay follows directly from the assumption on the almost-exponential decay of $w_{\gamma^{\prime}}(t)$ for $|t|$ large enough and, rapidly decaying Lieb-Robinson bounds in $r^{\prime}$, on the norm of the commutator:

$$
\left[H_{s}^{u}\left(r+r^{\prime}\right)-H_{s}^{u}\left(r+r^{\prime}-1\right), \tau_{t}^{H_{s}^{u}\left(r+r^{\prime}\right)}\left(O_{u}(r)\right)\right]
$$

for small enough $|t|$. In particular, note that for

$$
\delta_{s, t}\left(r^{\prime} ; O_{u}(r)\right)=\left\|\tau_{t}^{H_{s}^{u}\left(r+r^{\prime}\right)}\left(O_{u}(r)\right)-\tau_{t}^{H_{s}^{u}\left(r+r^{\prime}-1\right)}\left(O_{u}(r)\right)\right\|,
$$

we have:

$$
\begin{aligned}
& \delta_{s, t}\left(r^{\prime} ; O_{u}(r)\right)=\left\|\tau_{-t}^{H_{s}^{u}\left(r+r^{\prime}-1\right)}\left(\tau_{t}^{H_{s}^{u}\left(r+r^{\prime}\right)}\left(O_{u}(r)\right)\right)-O_{u}(r)\right\| \\
& \leq \int_{0}^{|t|}\left\|\left[H_{s}^{u}\left(r+r^{\prime}\right)-H_{s}^{u}\left(r+r^{\prime}-1\right), \tau_{t^{\prime}}^{H_{s}^{u}\left(r+r^{\prime}\right)}\left(O_{u}(r)\right)\right]\right\| d t^{\prime} .
\end{aligned}
$$


Defining $I_{q}(u)=\left\{Z \subset b_{u}(q): Z \cap\left(b_{u}(q) \backslash b_{u}(q-1)\right) \neq \emptyset\right\}$, we continue the above estimate as follows:

$$
\begin{aligned}
\delta_{s, t}\left(r^{\prime} ; O_{u}(r)\right) \leq & \sum_{b_{v}(1) \in I_{r+r^{\prime}}(u)} \int_{0}^{|t|}\left\|\left[Q_{v}, \tau_{t^{\prime}}^{H_{s}^{u}\left(r+r^{\prime}\right)}\left(O_{u}(r)\right)\right]\right\| d t^{\prime} \\
& +\sum_{b_{v}(k) \in I_{r+r^{\prime}}(u)} \int_{0}^{|t|} s\left\|\left[V_{v}(k), \tau_{t^{\prime}}^{H_{s}^{u}\left(r+r^{\prime}\right)}\left(O_{u}(r)\right)\right]\right\| d t^{\prime} .
\end{aligned}
$$

Note that the norm of the commutator $\left\|\left[Q_{v}, \tau_{t^{\prime}}^{H_{s}^{u}\left(r+r^{\prime}\right)}\left(O_{u}(r)\right)\right]\right\|$ can be bounded directly using Lieb-Robinson bounds for the evolution $\tau_{t^{\prime}}^{H_{s}^{u}\left(r+r^{\prime}\right)}$, with rapid decay in the distance between the supports of $b_{v}(1) \in I_{r+r^{\prime}}(u)$ and $b_{u}(r)$, which is at least $r^{\prime}-4$. Similarly, $\left\|\left[V_{v}(k), \tau_{t^{\prime}}^{H_{s}^{u}\left(r+r^{\prime}\right)}\left(O_{u}(r)\right)\right]\right\|$ will decay rapidly in the distance between $b_{v}(k) \in I_{r+r^{\prime}}(u)$ and $b_{u}(r)$, which is at least $r^{\prime}-2 k$. Of course, for $k \geq r^{\prime} / 2$ we may use the simple bound $\left\|\left[V_{v}(k), \tau_{t^{\prime}}^{H_{s}^{u}\left(r+r^{\prime}\right)}\left(O_{u}(r)\right)\right]\right\| \leq$ $2\left\|V_{v}(k)\right\|\left\|O_{u}(r)\right\| \leq 2 f_{0}(k)\left\|O_{u}(r)\right\|$. On the other hand, we also have the trivial bound for all $t \in \mathbb{R}:$

$$
\delta_{s, t}\left(r^{\prime} ; O_{u}(r)\right) \leq\left\|\tau_{t}^{H_{s}^{u}\left(r+r^{\prime}\right)}\left(O_{u}(r)\right)\right\|+\left\|\tau_{t}^{H_{s}^{u}\left(r+r^{\prime}-1\right)}\left(O_{u}(r)\right)\right\|=2\left\|O_{u}(r)\right\| .
$$

Combining the above bounds with the usual technique of bounding the integral:

$$
\int_{-\infty}^{\infty} f(t) \cdot g(t) d t \leq\|g\| \int_{-T}^{T}|f(t)| d t+\|f\| \int_{|t| \geq T}|g(t)| d t
$$

where $\|f\|$ is the supremum of $f$ over the appropriate domain, gives us the fastest decay for the optimal choice of the cut-off time $T$. In our case, $f(t)=\delta_{s, t}\left(r^{\prime} ; O_{u}(r)\right)$ and $g(t)=w_{\gamma^{\prime}}(t)$.

Now, for the final statement of the Lemma, we note that Duhamel's differentiation formula implies:

$$
\begin{aligned}
& \mathcal{F}_{\gamma^{\prime}, s}\left(Q_{u}\right)-\mathcal{F}_{\gamma^{\prime}, 0}\left(Q_{u}\right)=\int_{-\infty}^{\infty} d t w_{\gamma^{\prime}}(t) \int_{0}^{s} \partial_{s^{\prime}} \tau_{t}^{H_{s^{\prime}}}\left(Q_{u}\right) d s^{\prime}= \\
& \int_{-\infty}^{\infty} d t w_{\gamma^{\prime}}(t) \int_{0}^{s} i\left[\int_{0}^{t} \tau_{t^{\prime}}^{H_{s^{\prime}}}(V) d t^{\prime}, \tau_{t}^{H_{s^{\prime}}}\left(Q_{u}\right)\right] d s^{\prime}= \\
& \int_{0}^{s} d s^{\prime} \int_{-\infty}^{\infty} d t w_{\gamma^{\prime}}(t) \tau_{t}^{H_{s^{\prime}}}\left(\int_{0}^{t} i\left[\tau_{-t^{\prime}}^{H_{s^{\prime}}}(V), Q_{u}\right] d t^{\prime}\right),
\end{aligned}
$$

where we performed a change of variables and used the linearity of the commutator to get the last line. Note that in the last line above, we have an evolution $\tau_{t}^{H_{s^{\prime}}}$ applied to an integral involving the commutator:

$$
\left[\tau_{-t^{\prime}}^{H_{s^{\prime}}}(V), Q_{u}\right]=\sum_{(v, k)}\left[\tau_{-t^{\prime}}^{H_{s^{\prime}}}\left(V_{v}(k)\right), Q_{u}\right]
$$

It should be clear at this point that by applying the "telescoping sum" procedure, described earlier in this proof, to the evolution $\tau_{-t^{\prime}}^{H_{s^{\prime}}}$, each commutator in the above sum is itself a sum of terms supported on larger and larger sets, with decreasing norm. To see this, note that we may bound the norm of each such term trivially:

$$
\left\|\left[\tau_{-t^{\prime}}^{H_{s^{\prime}}^{v}\left(k+k^{\prime}\right)}\left(V_{v}(k)\right)-\tau_{-t^{\prime}}^{H_{s^{\prime}}^{v}\left(k+k^{\prime}-1\right)}\left(V_{v}(k)\right), Q_{u}\right]\right\| \leq 2 \delta_{s^{\prime},-t^{\prime}}\left(k^{\prime} ; V_{v}(k)\right)\left\|Q_{u}\right\|
$$

using the appropriate bounds on $\delta_{s^{\prime},-t^{\prime}}\left(k^{\prime} ; V_{v}(k)\right)$, as described above. In all cases, we note that the commutator vanishes for $k+k^{\prime} \leq d(v, u)-3$, where $d(v, u)$ is the distance between the sites $u, v \in \Lambda$, so we only need to consider $k^{\prime} \geq \max \{0, d(u, v)-k-2\}$. Finally, after localizing the terms inside the evolution $\tau_{t}^{H_{s^{\prime}}}$, we perform another "telescoping sum" decomposition on this final evolution, arriving at the final decomposition of strength $J$ (because of the norm of the terms $\left.V_{v}(k)\right)$ and decay given by a function $f_{1}^{(1)}$, whose decay depends implicitly on the decay of $w_{\gamma^{\prime}}$ and Lieb-Robinson bounds corresponding to the unitary evolutions under Hamiltonians with interactions coming from $Q_{u}, u \in \Lambda$ and $V_{v}(k), v \in \Lambda, k \geq 0$. The linear dependence of the bound on $s$ follows from the triangle inequality:

$$
\left\|\int_{0}^{s} d s^{\prime} A\left(s^{\prime}\right)\right\| \leq s \cdot \sup _{s^{\prime} \in[0, s]}\left\|A\left(s^{\prime}\right)\right\| .
$$




\section{B. Absorbing the perturbation.}

In this subsection, we focus on unitarily transforming the Hamiltonian $H_{s}=H_{0}+s V$, satisfying the conditions of Definition 4 into a Hamiltonian $H_{s}^{\prime}=H_{0}+V_{s}^{\prime}+E_{s} \mathbb{1}$, with the new perturbation $V_{s}^{\prime}$ satisfying certain stronger conditions with respect to the local ground states of $H_{0}$, as discussed at the beginning of this section. Since the whole process is a unitary transformation combined with a global energy shift, the spectral gap of $H_{s}$ is identical to that of $H_{0}+V_{s}^{\prime}$. The transformation is done in four steps:

1. We transform the terms $\mathcal{F}_{\gamma^{\prime}, s}^{u}:=\mathcal{F}_{\gamma^{\prime}, s}\left(Q_{u}\right)+s \mathcal{F}_{\gamma^{\prime}, s}\left(V_{u}\right)$ of $H_{s}=\sum_{u} \mathcal{F}_{\gamma^{\prime}, s}^{u}$, satisfying $\left[\mathcal{F}_{\gamma^{\prime}, s}^{u}, P_{0}(s)\right]=0$, into terms $X_{u}=U(s)^{\dagger} \mathcal{F}_{\gamma^{\prime}, s}^{u} U(s)-\mathcal{F}_{\gamma^{\prime}, 0}\left(Q_{u}\right)$, satisfying:

$$
\left[X_{u}, P_{0}\right]=U(s)^{\dagger}\left[\mathcal{F}_{\gamma^{\prime}, s}^{u}, P_{0}(s)\right] U(s)-\left[\mathcal{F}_{\gamma^{\prime}, 0}\left(Q_{u}\right), P_{0}\right]=0,
$$

where the unitary $U(s)$ satisfies $U(s) P_{0} U(s)^{\dagger}=P_{0}(s)$. At this point, the Hamiltonian $H_{s}$ has been unitarily transformed into $H_{s}^{\prime}:=U(s)^{\dagger} H_{s} U(s)=H_{0}+\sum_{u} X_{u}$.

2. We decompose $X_{u}=X_{u}^{(1)}+X_{u}^{(2)}+X_{u}^{(3)}$, where:

$$
X_{u}^{(1)}=\mathcal{F}_{\gamma^{\prime}, s}\left(Q_{u}\right)-\mathcal{F}_{\gamma^{\prime}, 0}\left(Q_{u}\right), \quad X_{u}^{(2)}=U(s)^{\dagger} \mathcal{F}_{\gamma^{\prime}, s}\left(Q_{u}\right) U(s)-\mathcal{F}_{\gamma^{\prime}, s}\left(Q_{u}\right), \quad X_{u}^{(3)}=s U(s)^{\dagger} \mathcal{F}_{\gamma^{\prime}, s}\left(V_{u}\right) U(s),
$$

and show that each term is a strength $J$ perturbation with decay given by functions $f_{1}^{(1)}, f_{1}^{(2)}$ and $f_{1}^{(3)}$, respectively, using Lemma 1 and properties of the unitary $U(s)$ [3, 17.

3. Using $\left[X_{u}, P_{0}\right]=0$, we rewrite $X_{u}=\left(1-P_{0}\right) \tilde{X}_{u}\left(1-P_{0}\right)+\Delta_{u}+c\left(X_{u}\right) \mathbb{1}$, where $\tilde{X}_{u}=X_{u}-c\left(X_{u}\right) \mathbb{1}, \Delta_{u}=P_{0} \tilde{X}_{u} P_{0}$ and $c\left(X_{u}\right)=\operatorname{Tr}\left(P_{0} X_{u}\right) / \operatorname{Tr} P_{0}$. We show $\left\|\Delta_{u}\right\|$ decays rapidly in $L^{*}$, using Local-TQO and the decomposition of $X_{u}$ from the previous step.

4. For a $\left(J, f_{1}\right)$ perturbation $X_{u}$ satisfying $\left[X_{u}, P_{0}\right]=0$, we prove that $W_{u}:=\left(1-P_{0}\right) \tilde{X}_{u}\left(1-P_{0}\right)$ is a $(J, w)$ perturbation, satisfying $W_{u}(r) P_{b_{u}(r)}=0$, with the decay $w(r)$ closely related to $f_{1}(r)$.

Note that throughout the transformation process, the perturbations $X_{u}, W_{u}$ and $\Delta_{u}$ depend implicitly on the parameter $s$. Nevertheless, the locality and bounds on the strength of the perturbations are independent of $s$ (some bounds have a linear dependence on $s$, but since $s \in[0,1]$, this makes the bounds only stronger.)

We state the end result of the above process as a Proposition:

Proposition 1. Let $H_{s}$ be the family of Hamiltonians considered in Definition 4 Then, there exist unitaries $U(s)$, such that: $U(s)^{\dagger} H_{s} U(s)=H_{0}+W+\Delta+E_{0}(s) \mathbb{1}$, with $W$ a $(J, w)$-perturbation satisfying, $W_{u}(r) P_{b_{u}(r)}=0,\|\Delta\|$ rapidly decaying in $L$ and $E_{0}(s)=\operatorname{Tr}\left(H_{s} P_{0}(s)\right) / \operatorname{Tr} P_{0}(s)$.

Proof. Using Lemma 1, we see that the first step implies $H_{s}^{\prime}:=U(s)^{\dagger} H_{s} U(s)=U(s)^{\dagger} \mathcal{F}_{\gamma^{\prime}, s}\left(H_{s}\right) U(s)=\mathcal{F}_{\gamma^{\prime}, 0}\left(H_{0}\right)+$ $\sum_{u} X_{u}=H_{0}+\sum_{u} X_{u}$, where $\left[X_{u}, P_{0}\right]=0$. The second and third step imply:

$$
H_{s}^{\prime}=H_{0}+\sum_{u}\left(1-P_{0}\right) \tilde{X}_{u}\left(1-P_{0}\right)+\Delta+c(X) \mathbb{1},
$$

for $\Delta=\sum_{u} \Delta_{u}$ a vanishingly small perturbation and $X=\sum_{u} X_{u}$. Noting that $X=U(s)^{\dagger} H_{s} U(s)-H_{0}$ and $P_{0}(s)=U(s) P_{0} U(s)^{\dagger}$, the cyclicity of the trace implies:

$$
c(X)=\operatorname{Tr}\left(H_{s} U(s) P_{0} U(s)^{\dagger}\right) / \operatorname{Tr} P_{0}-\operatorname{Tr}\left(H_{0} P_{0}\right) / \operatorname{Tr} P_{0}=\operatorname{Tr}\left(H_{s} P_{0}(s)\right) / \operatorname{Tr} P_{0}(s),
$$

so $H_{s}^{\prime}=H_{0}+\left(1-P_{0}\right) \tilde{X}\left(1-P_{0}\right)+\Delta+E_{0}(s) \mathbb{1}$. Finally, setting $W=\sum_{u} W_{u}$ and using the second and fourth steps, we get the desired result.

We focus, now, on proving the last three steps above, since the first step follows immediately from the properties of $\mathcal{F}_{\gamma^{\prime}, s}$ and the quasi-adiabatic evolution $U(s)$ (see, e.g. [3, Prop. 2.4].) For the second step above, we make extensive use of results obtained in [3, 17. We highly recommend reading through [17 to get a clear conceptual understanding of the ideas behind Hastings' quasi-adiabatic evolution $U(s)$ and its many applications to problems in condensed matter physics and quantum information theory. Below, we reference results from [3], from which one may extract explicit bounds on the decay of $X_{u}^{(2)}$ and $X_{u}^{(3)}$. The properties of $X_{u}^{(1)}$ follow directly from Lemma 1 . 
Lemma 2. Let $H_{s}$ be the family of gapped Hamiltonians described in Definition 4 and define $\mathcal{G}_{\gamma^{\prime}, s}\left(O_{u}(r)\right):=$ $\alpha_{s}^{\Lambda}\left(O_{u}(r)\right)$, where $\alpha_{s}^{\Lambda}$ is defined in [3] as the spectral flow corresponding to $H_{s}$ and $O_{u}(r)$ is an operator supported on $b_{u}(r)$. Then,

$$
\mathcal{G}_{\gamma^{\prime}, s}\left(O_{u}(r)\right)=\sum_{r^{\prime}=r_{0}}^{L-r} \mathcal{G}_{\gamma^{\prime}, s}\left(r^{\prime} ; O_{u}(r)\right), \quad r_{0} \geq 0,
$$

with $\mathcal{G}_{\gamma^{\prime}, s}\left(r^{\prime} ; O_{u}(r)\right)$ supported on $b_{u}\left(r+r^{\prime}\right)$ and

$$
\begin{aligned}
\left\|\mathcal{G}_{\gamma^{\prime}, s}\left(r^{\prime} ; O_{u}(r)\right)\right\| & \leq J\left\|O_{u}(r)\right\| g\left(r^{\prime}\right), \quad r^{\prime}>r_{0}, \\
\left\|\mathcal{G}_{\gamma^{\prime}, s}\left(r_{0} ; O_{u}(r)\right)-O_{u}(r)\right\| & \leq s J\left\|O_{u}(r)\right\| g\left(r_{0}\right), \quad\left\|\mathcal{G}_{\gamma^{\prime}, s}\left(r_{0} ; O_{u}(r)\right)\right\|=\left\|O_{u}(r)\right\|,
\end{aligned}
$$

for a rapidly decaying function $g$.

Proof. We introduce the decomposition $\mathcal{G}_{\gamma^{\prime}, s}\left(O_{u}(r)\right)=\sum_{r^{\prime}=r_{0}}^{L-r} \mathcal{G}_{\gamma^{\prime}, s}\left(r^{\prime} ; O_{u}(r)\right)$ :

$$
\begin{aligned}
& \mathcal{G}_{\gamma^{\prime}, s}\left(r^{\prime} ; O_{u}(r)\right)=\alpha_{s}^{\Lambda_{r^{\prime}}}\left(O_{u}(r)\right)-\alpha_{s}^{\Lambda_{r^{\prime}-1}}\left(O_{u}(r)\right), \quad r^{\prime} \geq r_{0}+1, \\
& \mathcal{G}_{\gamma^{\prime}, s}\left(r_{0} ; O_{u}(r)\right)=\alpha_{s}^{\Lambda_{r_{0}}}\left(O_{u}(r)\right) \quad r_{0} \geq 0,
\end{aligned}
$$

where we have defined $\Lambda_{n}=b_{u}(r+n), n \geq 0$ and used Eq. (4.42-4.46) of 3 to define $\alpha_{s}^{Y}(A)=U_{Y}^{\dagger}(s) A U_{Y}(s)$ :

$$
\partial_{s} U_{Y}(s)=i D_{Y}(s) U_{Y}(s), \quad U_{Y}(0)=\mathbb{1}, \quad D_{Y}(s)=\sum_{Z \subset Y} \Psi_{\Lambda}(Z, s) .
$$

First, notice that since each term $\Psi_{\Lambda}(Z, s)$ is supported on $Z$ by definition (see [3]), the terms $\mathcal{G}_{\gamma^{\prime}, s}\left(r^{\prime} ; O_{u}(r)\right)$ have support on $b_{u}\left(r+r^{\prime}\right)$, as desired. Moreover, since $\mathcal{G}_{\gamma^{\prime}, s}\left(r_{0} ; O_{u}(r)\right)=\alpha_{s}^{\Lambda_{r_{0}}}\left(O_{u}(r)\right)=U_{\Lambda_{r_{0}}}^{\dagger}(s) O_{u}(r) U_{\Lambda_{r_{0}}}(s)$, we immediately get $\left\|\mathcal{G}_{\gamma^{\prime}, s}\left(r_{0} ; O_{u}(r)\right)\right\|=\left\|O_{u}(r)\right\|$. To bound the norm of $\mathcal{G}_{\gamma^{\prime}, s}\left(r^{\prime} ; O_{u}(r)\right)$ for $r^{\prime}>r_{0}$, we need to bound $\left\|\alpha_{s}^{\Lambda_{r^{\prime}}}\left(O_{u}(r)\right)-\alpha_{s}^{\Lambda_{r^{\prime}-1}}\left(O_{u}(r)\right)\right\|$. As we show in Appendix A the norm $\left\|\alpha_{s}^{\Lambda_{r^{\prime}}}\left(O_{u}(r)\right)-\alpha_{s}^{\Lambda_{r^{\prime}-1}}\left(O_{u}(r)\right)\right\|$ is bounded above by:

$$
J\left\|O_{u}(r)\right\| g\left(r^{\prime}\right),
$$

for a rapidly decaying function $g$. A similar estimate shows that:

$$
\left\|\mathcal{G}_{\gamma^{\prime}, s}\left(r_{0} ; O_{u}(r)\right)-O_{u}(r)\right\| \leq \int_{0}^{s} d s^{\prime}\left\|\left[D_{\Lambda_{r_{0}}}\left(s^{\prime}\right), \alpha_{s}^{\Lambda_{r_{0}}}\left(O_{u}(r)\right)\right]\right\| \leq s J\left\|O_{u}(r)\right\| g\left(r_{0}\right) .
$$

The third step makes use of the Local-TQO condition, allowing us to extract the groundstate energy of $H_{s}$ as a single quantity $E_{0}(s)$, up to a small correction. In other words, the following Lemma asserts the vanishingly small splitting of the groundstate subspace for the gapped Hamiltonians $H_{s}$.

Lemma 3. Let $X_{u}$ be a $\left(J, f_{1}\right)$-perturbation centered on $u \in \Lambda$. Then, Assumption 1 implies for $\Delta_{u}=P_{0} \tilde{X}_{u} P_{0}$, $\tilde{X}_{u}=X_{u}-c\left(X_{u}\right) \mathbb{1}, c\left(X_{u}\right)=\operatorname{Tr}\left(X_{u} P_{0}\right) / \operatorname{Tr} P_{0}:$

$$
\left\|\Delta_{u}\right\| \leq J\left(\sum_{r=0}^{L^{*}} f_{1}(r) \Delta_{0}(L-r)+2 \sum_{r>L^{*}}^{L} f_{1}(r)\right) .
$$

Proof. By assumption, $X_{u}=\sum_{r \geq 0} X_{u}(r)$, with $X_{u}(r)$ supported on $b_{u}(r)$ and $\left\|X_{u}(r)\right\| \leq J f_{1}(r)$. We introduce an energy shift by redefining the local terms:

$$
\tilde{X}_{u}(r):=X_{u}(r)-c\left(X_{u}(r)\right) \mathbb{1}, \quad c\left(X_{u}(r)\right)=\operatorname{Tr}\left(P_{0} X_{u}(r)\right) / \operatorname{Tr} P_{0} .
$$

From Local-TQO we know that for $r \leq L^{*}$,

$$
\left\|P_{0} \tilde{X}_{u}(r) P_{0}\right\| \leq\left\|X_{u}(r)\right\| \Delta_{0}(L-r) .
$$


On the other hand, for $r>L^{*}$, we have:

$$
\left\|P_{0} \tilde{X}_{u}(r) P_{0}\right\| \leq\left\|X_{u}(r)\right\|+\left|c\left(X_{u}(r)\right)\right| \leq 2 J f_{1}(r) .
$$

Using (37) and (38), we get the desired bound:

$$
\left\|P_{0} \tilde{X}_{u} P_{0}\right\| \leq J\left(\sum_{r=0}^{L^{*}} f_{1}(r) \Delta_{0}(L-r)+2 \sum_{r>L^{*}}^{L} f_{1}(r)\right) .
$$

We now move to the last step of our transformation.

Lemma 4. Consider a frustration-free Hamiltonian $H_{0}$ and a perturbation $X_{u}$ with strength $J$ and decay $f_{1}(r)$, satisfying $\left[X_{u}, P_{0}\right]=0$. Set $W_{u}=\left(1-P_{0}\right) \tilde{X}_{u}\left(1-P_{0}\right)$, where $\tilde{X}_{u}=X_{u}-c\left(X_{u}\right) \mathbb{1}$. Then, $W_{u}$ is a strength $J$ perturbation with decay $w(r)$ given below and $W_{u}(r) P_{b_{u}(r)}=0$.

Proof. By definition, $W_{u} P_{0}=0$. Moreover, using $\left[X_{u}, P_{0}\right]=0$, we have: $\tilde{X}_{u}=W_{u}+\Delta_{u}$, where $\Delta_{u}=P_{0} \tilde{X}_{u} P_{0}$. This implies that:

$$
W_{u}=\sum_{r \geq 0}^{L-1} \tilde{X}_{u}(r)+\left(\tilde{X}_{u}(L)-\Delta_{u}\right) .
$$

Let us choose $\ell \leq L^{*}$. We apply Corollary 2 to $\sum_{r=0}^{\ell} \tilde{X}_{u}(r)$ :

$$
\begin{aligned}
& \left\|\sum_{r=0}^{\ell} \tilde{X}_{u}(r) P_{b_{u}(2 \ell)}\right\| \leq\left\|\sum_{r=0}^{\ell} \tilde{X}_{u}(r) P_{0}\right\|+2\left\|\sum_{r=0}^{\ell} \tilde{X}_{u}(r)\right\| \sqrt{\Delta_{0}(\ell)} \\
& \leq \underbrace{\left\|W_{u} P_{0}\right\|}_{0}+\left\|\sum_{r>\ell}^{L} \tilde{X}_{u}(r) P_{0}\right\|+\left\|\Delta_{u}\right\|+2\left\|\sum_{r=0}^{\ell} \tilde{X}_{u}(r)\right\| \sqrt{\Delta_{0}(\ell)} \\
& \leq 2 J\left(\sum_{r>\ell}^{L} f_{1}(r)+2 \sum_{r=0}^{\ell} f_{1}(r) \sqrt{\Delta_{0}(\ell)}\right)+\left\|\Delta_{u}\right\|,
\end{aligned}
$$

where we used $\left\|\tilde{X}_{u}(r)\right\| \leq 2\left\|X_{u}(r)\right\|$. We turn our attention to the final decomposition of $W_{u}$ into interaction terms that are annihilated by local ground states.

Define an orthogonal unity decomposition $\mathbb{1}=\sum_{m=1}^{L+1} E_{m}$, as in [6]:

$$
E_{m}=P_{b_{u}(m-1)}-P_{b_{u}(m)}, \quad 1 \leq m \leq L+1, \quad P_{u}=\mathbb{1}, \quad P_{b_{u}(L+1)}=0 .
$$

Using $\sum_{1 \leq p, r \leq L}=\sum_{j \geq 2}^{2 L} \sum_{p+r=j}$ and $\sum_{r \leq p \leq s} E_{p}=P_{b_{u}(r-1)}-P_{b_{u}(s)}$, we get:

$$
W_{u}:=\left(1-P_{0}\right) \tilde{X}_{u}\left(1-P_{0}\right)=\sum_{j \geq 2}^{L-1} Y_{u}(j)+Y_{u}(L)+\sum_{2 q=2}^{L} Z_{u}(2 q-1)+Z_{u}(L),
$$

where we defined:

$$
\begin{aligned}
& Y_{u}(j):=\sum_{p+r=j} E_{p} \sum_{q=0}^{\ell(p, r)} \tilde{X}_{u}(q) E_{r}, \quad 2 \leq j \leq L-1, \\
& Y_{u}(L):=\sum_{p+r=L}^{2 L} E_{p} \sum_{q=0}^{\ell(p, r)} \tilde{X}_{u}(q) E_{r}, \quad \ell(p, r):=\lfloor\max (p, r) / 2\rfloor, \\
& Z_{u}(2 q-1):=\left(\mathbb{1}-P_{b_{u}(2 q-1)}\right) \tilde{X}_{u}(q)\left(\mathbb{1}-P_{b_{u}(2 q-1)}\right), \quad 2 \leq 2 q \leq L, \\
& Z_{u}(L):=\left(1-P_{0}\right) \sum_{q>L / 2} \tilde{X}_{u}(q)\left(1-P_{0}\right) .
\end{aligned}
$$


Note that $Y_{u}(j) P_{b_{j}(u)}=0$ and $Z_{u}(2 q-1) P_{b_{u}(2 q-1)}=0$. In other words, $Y_{u}(j)$ and $Z_{u}(2 q-1)$ are supported on $b_{j}(u)$ and $b_{u}(2 q-1)$, respectively, and are annihilated by the local ground states. The same holds for the terms $Y_{u}(L)$ and $Z_{u}(L)$. Finally:

$$
\begin{aligned}
& \left\|Z_{u}(2 q-1)\right\| \leq\left\|\tilde{X}_{u}(q)\right\| \leq 2 J f_{1}(q), \quad\left\|Z_{u}(L)\right\| \leq 2 J \sum_{q>L / 2} f_{1}(q), \\
& \left\|Y_{u}(j)\right\| \leq \sum_{p+r=j}\left\|E_{p} \sum_{q=0}^{\ell(p, r)} \tilde{X}_{u}(q) E_{r}\right\| \\
& \leq 2 \sum_{p \geq j-p}\left\|E_{p} \sum_{q=0}^{\lfloor p / 2\rfloor} \tilde{X}_{u}(q)\right\| \leq 2 \sum_{p \geq j-p}\left\|P_{b_{u}(p-1)} \sum_{q=0}^{\lfloor p / 2\rfloor} \tilde{X}_{u}(q)\right\| \\
& \leq 2 J \sum_{p>\lfloor j / 2\rfloor}^{\lfloor-1}\left(\sum_{r \geq\lfloor p / 2\rfloor}^{L} f_{1}(r)+2 \sum_{r=0}^{L p / 2\rfloor} f_{1}(r) \sqrt{\Delta_{0}(p)}\right)+j\left\|\Delta_{u}\right\|,
\end{aligned}
$$

where the last line follows from 43 . To bound the norm of $Y_{u}(L)$, we simply sum over the bounds for the norms of $Y_{u}(j)$ :

$$
\left\|Y_{u}(L)\right\| \leq 2 J \sum_{j \geq L}^{2 L} \sum_{p>\lfloor j / 2\rfloor}^{j-1}\left(\sum_{r \geq\lfloor p / 2\rfloor}^{L} f_{1}(r)+2 \sum_{r=0}^{\lfloor p / 2\rfloor} f_{1}(r) \sqrt{\Delta_{0}(p)}\right)+2 L^{2}\left\|\Delta_{u}\right\| .
$$

We define the function $w(r)$ as follows:

$$
\begin{aligned}
& w(r)=\left\|Y_{u}(r)\right\|, \quad r \equiv 0 \quad(\bmod 2), \quad r<L, \\
& w(r)=\left\|Y_{u}(r)\right\|+\left\|Z_{u}(r)\right\|, \quad r \equiv 1 \quad(\bmod 2) \quad r<L, \\
& w(L)=\left\|Y_{u}(L)\right\|+\left\|Z_{u}(L)\right\| .
\end{aligned}
$$

For $\Delta_{0}(r)$ and $f_{1}(r)$ decaying rapidly, we see from 44,48 and Lemma 3 that $w(r)$ is also rapidly decaying.

\section{PROOF OF RELATIVE BOUNDEDNESS}

We now generalize the proof of Proposition 1 in [6].

Proposition 2. Let $W$ be a $(J, w)$-perturbation satisfying: $W_{u}(r) P_{b_{u}(r)}=0$. For $H_{0}$ a frustration-free, locally-gapped Hamiltonian on $\Lambda \subset \mathbb{Z}^{d}$, with gap decay $\gamma(r)$, there exists a constant

$$
c:=C_{d} \sum_{k=1}^{M} \frac{r_{k}^{d}}{\gamma\left(r_{k}\right)} \sum_{r>r_{k-1}}^{r_{k}} w(r), \quad 0=r_{0}<r_{1} \leq \ldots \leq r_{M}=L
$$

where $C_{d}$ is the volume of the unit ball in $\mathbb{Z}^{d}$, such that for arbitrary states $|\psi\rangle$ :

$$
|\langle\psi|W| \psi\rangle| \leq c \cdot J\left\langle\psi\left|H_{0}\right| \psi\right\rangle
$$

Proof. First, we partition the lattice $\Lambda$ into disjoint sets $\mathcal{C}_{j}(r), 1 \leq j \leq N(r)$, such that any two distinct points $u, v \in C_{j}(r)$ satisfy $b_{u}(r) \cap b_{v}(r)=\emptyset$. We treat the case $8 r>L$ separately, using the trivial decomposition $C_{j}(r)=$ $\left\{u_{j}\right\}, u_{j} \in \Lambda, 1 \leq j \leq N(r)=L^{d}$. To accomplish the decomposition for $8 r \leq L$, we start by dividing $\Lambda$ into an even number of boxes of size $2 r^{\prime}$, in each direction, such that $2 r \leq 2 r^{\prime} \leq 3 r$. In particular, we have $L=2 r q+r_{0}, 0 \leq r_{0}<2 r$, so we may choose $2 r^{\prime}=2 r q /(q-b)+r_{0} /(q-b)$, where $b \equiv q(\bmod 2)$. Since we assumed $8 r \leq L$, we know that $2 r \cdot 3+r_{0}<L \Longrightarrow q \geq 4$, which implies the bounds on $2 r^{\prime}$. Note that

$$
L / 2 r^{\prime}=q-b \equiv 0 \quad(\bmod 2)
$$

and that $r^{\prime}$ need not be an integer.

We build the partition one dimension at a time, as follows: In one dimension, we "color" every other interval of length $2 r^{\prime}$ on the line with the same color. So, in one dimension, we have 2 colors. In 2 dimensions, we fill the plane 


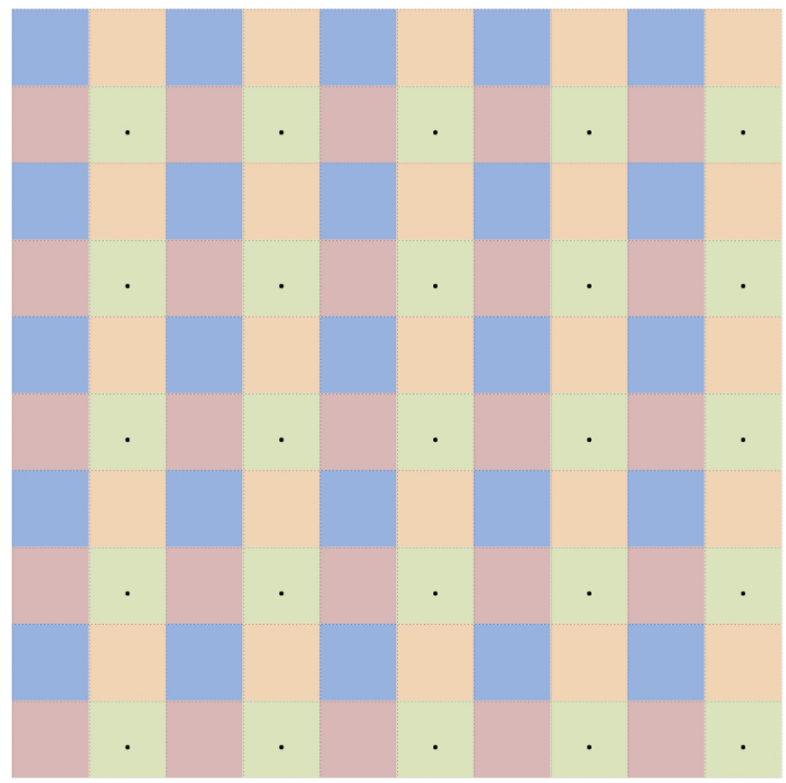

FIG. 1. Partition of the lattice $\Lambda$ into an even number of boxes of size $2 r^{\prime}$ each. The black dots indicate the points $u_{j}^{a}$ inside some set $C_{j}(r)$. The label $a$ corresponds to the label of the box in which the point is found.

with an even number of rows of $2 r^{\prime} \times 2 r^{\prime}$ squares, where every other row looks the same (same "color" pattern) and within each row we have an even number of alternating colored boxes (see Fig. 1.) We can continue like this for any dimension $d$ of our lattice, requiring $2^{d}$ colors in total. Now, because of the way we have constructed our partition, any two points on distinct boxes of the same color will be a distance at least $2 r^{\prime} \geq 2 r$ apart, as desired. By choosing exactly one point $u_{j}^{a}$ from each box $B_{a}$ of the same color, we get a family of disjoint sets $\mathcal{C}_{j}(r)$ that cover $\Lambda$, each satisfying the desired property of internal sparsity. The total number $N(r)$ of these sets is bounded by the number of colors multiplied by the volume of each box. So, we have:

$$
N(r) \leq 2^{d}\left(2 r^{\prime}\right)^{d} \leq 6^{d} r^{d}
$$

and

$$
\Lambda=\bigcup_{j \geq 1}^{N(r)} \mathcal{C}_{j}(r), \quad \text { with } \mathcal{C}_{j}(r)=\left\{u_{j}^{a}, a \geq 1\right\} \text { defined above. }
$$

From this point onward, we set:

$$
b_{j, a}(r):=b_{u_{j}^{a}}(r), \quad u_{j}^{a} \in \mathcal{C}_{j}(r), \quad a \geq 1 .
$$

Note that $\left[P_{b_{j, a}(r)}, P_{b_{j, b}(r)}\right]=0$, since the projectors have disjoint supports for $a \neq b$. Define an orthogonal decomposition of unity as follows:

$$
R_{j}^{\mathcal{Y}}(r)=\prod_{a=1}^{\left|\mathcal{C}_{j}(r)\right|}\left(\mathcal{Y}_{a}\left(1-P_{b_{j, a}(r)}\right)+\left(1-\mathcal{Y}_{a}\right) P_{b_{j, a}(r)}\right), \quad \mathcal{Y} \in\{0,1\}^{\left|\mathcal{C}_{j}(r)\right|} .
$$

It is easy to check that $\sum_{\mathcal{Y}} R_{j}^{\mathcal{Y}}(r)=\mathbb{1}$ and $R_{j}^{\mathcal{Y}}(r) R_{j}^{\mathcal{Z}}(r)=\delta_{\mathcal{Y}, \mathcal{Z}} R_{j}^{\mathcal{Y}}(r)$.

Before continuing, we define a decomposition of the perturbation $W$ as follows:

$$
\begin{array}{r}
W=\sum_{k \geq 1}^{M} \sum_{j \geq 1}^{N\left(r_{k}\right)} \hat{W}_{j}\left(r_{k}\right), \quad \hat{W}_{j}\left(r_{k}\right):=\sum_{a: u_{j}^{a} \in \mathcal{C}_{j}\left(r_{k}\right)} \hat{W}_{u_{j}^{a}}\left(r_{k}\right), \\
\hat{W}_{u}\left(r_{k}\right):=\sum_{r>r_{k-1}}^{r_{k}} W_{u}(r), \quad 0=r_{0}<r_{1} \leq r_{2} \leq \ldots \leq r_{M}=L .
\end{array}
$$


Since $W(r)$ is locally block-diagonal, we have

$$
W_{u_{j}^{a}}(r) P_{b_{j, a}(r)}=0 \Longrightarrow \hat{W}_{u_{j}^{a}}\left(r_{k}\right) P_{b_{j, a}\left(r_{k}\right)}=0 .
$$

Moreover, recalling that $w(r):=\sup _{u}\left\|W_{u}(r)\right\| / J$, we have:

$$
\left\|\hat{W}_{u_{j}^{a}}\left(r_{k}\right)\right\| \leq J \sum_{r>r_{k-1}}^{r_{k}} w(r) .
$$

Setting $\hat{w}\left(r_{k}\right):=\sum_{r>r_{k-1}}^{r_{k}} w(r)$, we will show that:

$$
\left|\left\langle\psi\left|\hat{W}_{j}\left(r_{k}\right)\right| \psi\right\rangle\right| \leq J \cdot \hat{w}\left(r_{k}\right)\left\langle\psi\left|G_{j}\left(r_{k}\right)\right| \psi\right\rangle,
$$

where,

$$
G_{j}\left(r_{k}\right):=\sum_{n \geq 1} n \cdot \sum_{|\mathcal{Y}|=n} R_{j}^{\mathcal{Y}}\left(r_{k}\right) .
$$

Define $\hat{W}_{j, a}\left(r_{k}\right):=\hat{W}_{u_{j}^{a}}\left(r_{k}\right)$, for $u_{j}^{a} \in \mathcal{C}_{j}\left(r_{k}\right)$. By definition of $R_{\mathcal{Y}}^{j}(r)$ and the local block-diagonality of $\hat{W}_{j}\left(r_{k}\right)=$ $\sum_{a} \hat{W}_{j, a}\left(r_{k}\right)$, we have:

$$
\begin{aligned}
& R_{j}^{\mathcal{Y}}\left(r_{k}\right) \hat{W}_{j}\left(r_{k}\right) R_{j}^{\mathcal{Z}}\left(r_{k}\right)=\delta \mathcal{Y}, \mathcal{Z} \sum_{a: \mathcal{Y}_{a}=1} R_{j}^{\mathcal{Y}}\left(r_{k}\right) \hat{W}_{j, a}\left(r_{k}\right) R_{j}^{\mathcal{Y}}\left(r_{k}\right) \Longrightarrow \\
& \left\|R_{j}^{\mathcal{Y}}\left(r_{k}\right) \hat{W}_{j}\left(r_{k}\right) R_{j}^{\mathcal{Z}}\left(r_{k}\right)\right\| \leq J \hat{w}\left(r_{k}\right) \delta \mathcal{Y}, \mathcal{Z} \cdot|\mathcal{Y}|,
\end{aligned}
$$

where the equality in the first line follows from the observations:

1. $\hat{W}_{j, a}\left(r_{k}\right) R_{j}^{\mathcal{Y}}\left(r_{k}\right)=0$, for $\mathcal{Y}_{a}=0$.

2. If $\mathcal{Y} \neq \mathcal{Z}$, then either $\mathcal{Z}_{a}=0$ when $\mathcal{Y}_{a}=1$ and $\hat{W}_{j, a}\left(r_{k}\right) R_{j}^{\mathcal{Z}}\left(r_{k}\right)=0$, or $\mathcal{Y}_{b}=0$ and $\mathcal{Z}_{b}=1$, for $b \neq a$, so $R_{j}^{\mathcal{Y}}\left(r_{k}\right) \hat{W}_{j, a}\left(r_{k}\right) R_{j}^{\mathcal{Z}}\left(r_{k}\right)$ equals:

$$
P_{b_{j, b}\left(r_{k}\right)}\left(1-P_{b_{j, b}\left(r_{k}\right)}\right) R_{j}^{\mathcal{Y}}\left(r_{k}\right) \hat{W}_{j, a}\left(r_{k}\right) R_{j}^{\mathcal{Z}}\left(r_{k}\right)=0 .
$$

The bound 61] implies for arbitrary states $|\psi\rangle$ :

$$
\begin{aligned}
& \left|\left\langle\psi\left|\hat{W}_{j}\left(r_{k}\right)\right| \psi\right\rangle\right| \leq \sum_{\mathcal{Y}}\left|\left\langle\psi\left|R_{j}^{\mathcal{Y}}\left(r_{k}\right) \hat{W}_{j}\left(r_{k}\right) R_{j}^{\mathcal{Y}}\left(r_{k}\right)\right| \psi\right\rangle\right| \\
& \leq \sum_{\mathcal{Y}}\left\|R_{j}^{\mathcal{Y}}\left(r_{k}\right) \hat{W}_{j}\left(r_{k}\right) R_{j}^{\mathcal{Y}}(r)\right\|\left\langle\psi\left|R_{j}^{\mathcal{Y}}\left(r_{k}\right)\right| \psi\right\rangle \leq J \hat{w}\left(r_{k}\right) \sum_{\mathcal{Y}}|\mathcal{Y}|\left\langle\psi\left|R_{j}^{\mathcal{Y}}\left(r_{k}\right)\right| \psi\right\rangle \\
& =J \hat{w}\left(r_{k}\right)\left\langle\psi\left|G_{j}\left(r_{k}\right)\right| \psi\right\rangle .
\end{aligned}
$$

Recalling that $H_{b_{j, a}(r)}=\sum_{u: b_{u}(1) \subset b_{j, a}(r)} Q_{u}$, define $H_{j}(r)=\sum_{a} H_{b_{j, a}(r)}$ and note that:

$$
\sum_{j=1}^{N(r)} H_{j}(r)=\sum_{u \in \Lambda}\left(\sum_{j \geq 1} \operatorname{Ind}\left(b_{u}(1) \subset \cup_{a \geq 1} b_{j, a}(r)\right)\right) Q_{u} \leq C_{d} r^{d} H_{0},
$$

where $C_{d}$ is the volume of the unit ball in $\mathbb{Z}^{d}$ and Ind denotes the indicator function. To see this, note that $\sum_{j \geq 1} \operatorname{Ind}\left(b_{u}(1) \subset \cup_{a \geq 1} b_{j, a}(r)\right)$ is bounded above by the number of balls of radius $r$ that contain $b_{u}(1)$, since $\operatorname{Ind}\left(b_{u}(1) \subset \cup_{a \geq 1} b_{j, a}(r)\right)$ is non-zero only when $j$ is such that $b_{j, a}(r):=b_{u_{j}^{a}}(r)$ includes $b_{u}(1)$ for some unique $a$, since $b_{j, a}(r) \cap b_{j, b}(r)=\emptyset$, for $a \neq b$. Thus, the indicator sum is bounded by the number of places we can put $b_{u}(1)$ inside a ball of radius $r$, which is the same as the number of balls of radius $r$ containing $b_{u}(1)$. The former is upper-bounded by the number of places we can put $b_{0}(u)=u$ inside a ball of radius $r$, which is exactly equal to $C_{d} r^{d}$.

Using the assumption that $H_{0}$ is locally gapped and frustration-free, we have

$$
H_{b_{j, a}(r)} \geq \gamma_{j, a}(r)\left(1-P_{b_{j, a}(r)}\right), \quad \mathcal{Y}_{a}=0 \Longrightarrow H_{b_{j, a}(r)} R_{\mathcal{Y}}^{j}(r)=0 .
$$


Then, we can proceed as follows, for arbitrary states $|\psi\rangle$ :

$$
\begin{aligned}
& \left\langle\psi\left|H_{j}(r)\right| \psi\right\rangle=\sum_{\mathcal{Y}} \sum_{a: \mathcal{Y}_{a}=1}\left\langle\psi\left|R_{j}^{\mathcal{Y}}(r) H_{b_{j, a}(r)} R_{j}^{\mathcal{Y}}(r)\right| \psi\right\rangle \\
& \geq \sum_{\mathcal{Y}} \sum_{a: \mathcal{Y}_{a}=1} \gamma_{j, a}(r)\left\langle\psi\left|R_{j}^{\mathcal{Y}}(r)\left(1-P_{b_{j, a}(r)}\right) R_{j}^{\mathcal{Y}}(r)\right| \psi\right\rangle \\
& \leq \gamma_{j}(r) \sum_{n \geq 1} n \sum_{|\mathcal{Y}|=n}\left\langle\psi\left|R_{j}^{\mathcal{Y}}(r)\right| \psi\right\rangle \Longrightarrow G_{j}\left(r_{k}\right) \leq H_{j}\left(r_{k}\right) / \gamma_{j}\left(r_{k}\right),
\end{aligned}
$$

where $\gamma_{j}(r):=\min _{a} \gamma_{j, a}(r)$. We have just shown that:

$$
\left|\left\langle\psi\left|\hat{W}_{j}\left(r_{k}\right)\right| \psi\right\rangle\right| \leq J \hat{w}\left(r_{k}\right)\left\langle\psi\left|G_{j}\left(r_{k}\right)\right| \psi\right\rangle \leq J \hat{w}\left(r_{k}\right) / \gamma_{j}\left(r_{k}\right)\left\langle\psi\left|H_{j}\left(r_{k}\right)\right| \psi\right\rangle .
$$

This implies that:

$$
|\langle\psi|W| \psi\rangle| \leq \sum_{k=1}^{M} \sum_{j=1}^{N\left(r_{k}\right)}\left|\left\langle\psi\left|\hat{W}_{j}\left(r_{k}\right)\right| \psi\right\rangle\right| \leq J \sum_{k=1}^{M} \hat{w}\left(r_{k}\right) \sum_{j=1}^{N\left(r_{k}\right)}\left\langle\psi\left|H_{j}\left(r_{k}\right)\right| \psi\right\rangle / \gamma_{j}\left(r_{k}\right) .
$$

Setting $\gamma(r):=\min _{j} \gamma_{j}(r)$, we have:

$$
|\langle\psi|W| \psi\rangle| \leq c J\left\langle\psi\left|H_{0}\right| \psi\right\rangle,
$$

where $c=C_{d} \sum_{k=1}^{M} r_{k}^{d} \hat{w}\left(r_{k}\right) / \gamma\left(r_{k}\right)$, using 52 and 62 .

We note a few things about the definition of the structural constant $c$ appearing in Proposition 2 (i) The choice of the $r_{k}: 0<r_{1} \leq \ldots \leq r_{M}=L$ is left unspecified beyond the boundary conditions, so that one may optimize the constant $c$ given the relevant decays $w(r)$ and $\gamma(r)$. For example, if $\gamma(r)$ decays particularly fast for some values of $r$, we may want to avoid these values by summing over them within the rapid decay of $w(r)$. (ii) Assuming polynomial decay of $\gamma\left(r_{k}\right)$ for the selected values of $r_{k}$, it is clear that for fast enough decay of $w(r)$ (superpolynomial, or even fast enough polynomial decay), $c$ is a constant depending only on the dimension $d$ of the lattice and the decay rate of $\gamma\left(r_{k}\right)$ and $w(r)$.

\section{FINAL ARGUMENT:}

The argument below follows the bootstrapping argument in [6], putting together all the pieces to prove stability of frustration-free systems under weak perturbations.

Theorem 1. Let $H_{0}$ be a frustration-free Hamiltonian, with spectral gap $\gamma$, defined in Section II, satisfying the Local$T Q O$ and Local-Gap conditions. For a $\left(J, f_{0}\right)$-perturbation $V$, with $f_{0}$ rapidly-decaying, there exists a constant $c$, given in Proposition 2, such that for $J \leq \frac{1}{3 c}$ and $L$ large enough, the spectral gap of $H_{0}+V$ is bounded from below by $\gamma / 2$.

Proof. Let $J_{0}=\frac{1}{3 c}$, where $c$ is defined in Proposition 2 . Assume that for $J \leq J_{0}, H_{0}+s V$ has gap $\gamma_{s} \geq \gamma / 2$, for $0 \leq s \leq s_{0}<1$ and $\gamma_{s}<\gamma / 2$, for $s_{0}<s \leq s_{1} \leq 1$. Then, we have proven in the previous sections that for $0 \leq s \leq s_{0}$ :

$$
U(s)^{\dagger}\left(H_{0}+s V\right) U(s)=H_{0}+W_{s}+\Delta_{s}+E_{s} \cdot \mathbb{1},
$$

where $U(s)$ generates the spectral flow $U(s) P_{0}(0) U(s)^{\dagger}=P_{0}(s)$, and $\left|\left\langle\psi\left|W_{s}\right| \psi\right\rangle\right| \leq c \cdot J\left\langle\psi\left|H_{0}\right| \psi\right\rangle$. Moreover, $E_{s} \cdot \mathbb{1}$ is an overall energy shift and the norm of $\Delta_{s}$ decays rapidly in $L$. We note at this point that the Local-TQO cut-off $L^{*}$ plays a crucial role in the decay estimates of $\left\|\Delta_{s}\right\|$, for example. In particular, $L^{*}$ should scale as $L^{\alpha}$, for some $\alpha \in(0,1]$, in order for estimates like the one in Lemma 3 to decay rapidly in $L$, especially since the estimate for $\left\|\Delta_{s}\right\|$ is given by $L^{d} \sup _{u \in \Lambda}\left\|\Delta_{u}\right\| \sim L^{d} \max \left\{\Delta_{0}\left(L^{*}\right), f_{0}\left(L^{*}\right)\right\}$.

We will show that, for large enough $L$, the gap of $H_{0}+W_{s_{0}}+\Delta_{s_{0}}$, which is $\gamma_{s_{0}}$, is strictly greater than $\gamma / 2$, contradicting the assumption that $\gamma_{s}<\gamma / 2$, for $s_{0}<s \leq s_{1}$, implying that $s_{0}=1$.

Now, we note that $H_{0}+W_{s}+\Delta_{s}=U(s)^{\dagger}\left(H_{0}+s V-E_{s} \cdot \mathbb{1}\right) U(s)$ has eigenstates in the subspace $P_{0}(0)$, with energy within $\left\|\Delta_{s}\right\|$ from 0 . Those states correspond to the "groundstate" subspace $P_{0}(s)$ of the unrotated version of the Hamiltonian, $H_{0}+s V$. In general, eigenstates of $H_{0}+s V$ in the $P_{0}(s)$ subspace will have different energies, but only 
up to vanishingly small error in the system size, $L$. To see this, note that for $\left|\Psi_{0}(s)\right\rangle=P_{0}(s)\left|\Psi_{0}(s)\right\rangle$ an eigenstate of $H_{0}+s V$ with energy $E_{0}(s)$, setting $\left|\Psi_{0}\right\rangle:=U(s)^{\dagger}\left|\Psi_{0}(s)\right\rangle$, we have $P_{0}(0)\left|\Psi_{0}\right\rangle=U(s)^{\dagger} P_{0}(s)\left|\Psi_{0}(s)\right\rangle=\left|\Psi_{0}\right\rangle$ and

$$
\left(H_{0}+W_{s}+\Delta_{s}\right)\left|\Psi_{0}\right\rangle=U^{\dagger}(s)\left(H_{0}+s V-E_{s} \cdot \mathbb{1}\right) U(s)\left|\Psi_{0}\right\rangle=\left(E_{0}(s)-E_{s}\right)\left|\Psi_{0}\right\rangle .
$$

Moreover, since $\left|\left\langle\Psi_{0}\left|W_{s}\right| \Psi_{0}\right\rangle\right| \leq c J\left\langle\Psi_{0}\left|H_{0}\right| \Psi_{0}\right\rangle=0$, the energy $E_{0}(s)$, satisfies

$$
\left(H_{0}+W_{s}+\Delta_{s}\right)\left|\Psi_{0}\right\rangle=\Delta_{s}\left|\Psi_{0}\right\rangle=E_{0}(s)\left|\Psi_{0}\right\rangle .
$$

Hence, $\left|E_{0}(s)\right| \leq\left\|\Delta_{s}\right\|<\gamma / 13$, for large enough $L$.

Moreover, any state $\left|\psi_{1}\right\rangle$ orthogonal to the $P_{0}(0)$ subspace has energy at least $(1-c J) \gamma-\left\|\Delta_{s}\right\|$, which follows from the linear relative bound on $W_{s}$ and the gap of $H_{0}$ :

$$
\begin{aligned}
\left\langle\psi_{1}\left|H_{0}+W_{s}+\Delta_{s}\right| \psi_{1}\right\rangle & \geq\left\langle\psi_{1}\left|H_{0}\right| \psi_{1}\right\rangle-\left|\left\langle\psi_{1}\left|W_{s}\right| \psi_{1}\right\rangle\right|-\left|\left\langle\psi_{1}\left|\Delta_{s}\right| \psi_{1}\right\rangle\right| \\
& \geq(1-c J)\left\langle\psi_{1}\left|H_{0}\right| \psi_{1}\right\rangle-\left\|\Delta_{s}\right\| \geq(1-c J) \gamma-\left\|\Delta_{s}\right\| .
\end{aligned}
$$

Now, taking the linear size $L$ large enough (such that $\left\|\Delta_{s}\right\| \leq \gamma / 13$ ) and recalling that $c J \leq 1 / 3$, we see that the gap is bounded below by:

$$
\gamma_{s} \geq(1-c J) \gamma-2\left\|\Delta_{s}\right\| \geq(20 / 39) \gamma>\gamma / 2
$$

for all $s \in\left[0, s_{0}\right]$. But, by definition of $s_{0}$, we know that for any $0<\epsilon \leq s_{1}-s_{0}$, we have $\gamma\left(s_{0}+\epsilon\right)<\gamma / 2$, a contradiction of the continuity of $\gamma_{s}$ for finite $L$. Hence, $s_{0}=1$ and $\gamma(1) \geq \gamma / 2$, for all $J \leq J_{0}$ and $L$ large enough to satisfy $\left\|\Delta_{s}\right\| \leq \gamma / 13$.

Finally, this implies that $H_{0}+s V$ has gap $\gamma_{s} \geq \gamma / 2$, for all $s \in[0,1]$ for the parameters of $J_{0}$ and $L$ given above and determined implicitly by the decay $f_{0}(r)$ of $V$, the Local-TQO decay $\Delta_{0}(r)$, the local gap $\gamma(r)$ and the linear size $L$ of the system.

\section{DISCUSSION}

We have shown that topological phases corresponding to the groundstate sector of gapped, frustration-free Hamiltonians are stable under quasi-local perturbations. In particular, the splitting in the energy of the groundstate subspace is bounded by a rapidly-decaying function of the system size and the gap to the high-energy sectors remains open for perturbation strengths independent of the system size. Our result also implies the stability of symmetry-protected sectors under perturbations that obey the symmetry of that sector. To see this, note that the Local-TQO condition is only required for operators $O_{A}$ obeying the symmetries under consideration. Stability follows using the methods already found here, as is already discussed in [6].

Moreover, it can be shown 35] that parent Hamiltonians of Matrix Product States (MPS) satisfy the Local-TQO condition with $\Delta_{0}$ decaying exponentially in these cases. Combined with the results of Nachtergaele [28] and Spitzer et al.42 on the spectral gap of one-dimensional, frustration-free Hamiltonians, our result shows that parent Hamiltonians of MPS have stable low-energy spectrum against arbitrary, sufficiently weak, quasi-local perturbations.

At this point, there are several important open questions that one may want to pursue:

1. First, can we extend the one-dimensional methods, introduced by Nachtergaele in [28], to prove rigorous spectral gaps for frustration-free systems in two dimensions?

2. Can we use the decay rate $\Delta_{0}$ in Local-TQO to classify different phases of matter? In other words, is there a canonical parent Hamiltonian construction, given a groundstate subspace, such that Local-TQO is satisfied with an optimal decay $\Delta_{0}$ ? For example, see [8, Sec. 2.4], where two different Hamiltonians for the toric code give very different decays $\Delta_{0}$; one being constant and the other being a step function.

3. Under what conditions (maybe translation invariance, and/or scale invariance) is Local-TQO satisfied, if the global groundstate space is topologically ordered? This is related to the previous question.

4. Can one rigorously prove that all gapped, frustration-free systems with non-vanishing, local interactions, satisfy the Local-Gap condition, using the detectability lemma, or some other method?

5. What features of parent Hamiltonians of PEPS can one use to prove that the Local-TQO and Local-Gap conditions are satisfied?

6. Can one use the present result to prove the Haldane conjecture, by identifying a gapped, frustration-free Hamiltonian which satisfies the Local-TQO and Local-Gap conditions, sufficiently close to the Haldane phase [23]? 
7. Can we identify two-dimensional, frustration-free systems satisfying Local-TQO that exhibit Topological Order at non-zero temperatures? What about three-dimensional frustration-free systems?

8. Finally, what is the form of Local-TQO that is truly necessary (and sufficient) for stability of the spectral gap against local perturbations, for frustrated, gapped Hamiltonians? Is the Local-TQO condition always true for generic gapped Hamiltonians?

\section{Acknowledgments:}

S. M. would like to thank Sergey Bravyi, Matt Hastings and Tobias Osborne for encouraging the pursuit of this result, Frank Verstraete for pointing out the connection of Local-TQO with the area-law for the entanglement entropy, Steve Flammia and Norbert Schuch for working out the details of that connection with the author, Robert Koenig and Bruno Nachtergaele for discussions on some of the technical details and Alexei Kitaev for several useful discussions on the extension of this result to general, gapped Hamiltonians. S.M. received support from NSF \#DMS-0757581 and \#PHY-0803371, and DOE Contract \#DE-AC52-06NA25396. S. M. and J. P. are grateful for the warm hospitality of Los Alamos National Lab, where part of this work was completed during the summer of 2010.

\section{Appendix A: Lieb-Robinson Estimates for Lemma 2}

We follow the proof of Thm. 5.2 in [3] to bound $\left\|\alpha_{s}^{b_{u}\left(r^{\prime}\right)}\left(O_{u}(r)\right)-\alpha_{s}^{b_{u}\left(r^{\prime}-1\right)}\left(O_{u}(r)\right)\right\|$ in Lemma 2. To use the notation of [3], we set $\Lambda_{n}=b_{u}(n+r)$, for $n \geq 0$, so that $O_{u}(r)$ is supported on the set $\Lambda_{0}$. We have:

$$
\begin{aligned}
& \left\|\alpha_{s}^{\Lambda_{r^{\prime}}}\left(O_{u}(r)\right)-\alpha_{s}^{\Lambda_{r^{\prime}-1}}\left(O_{u}(r)\right)\right\| \leq \int_{0}^{s}\left\|\left[D_{\Lambda_{r^{\prime}}}(t)-D_{\Lambda_{r^{\prime}-1}}(t), \alpha_{t}^{\Lambda_{r^{\prime}-1}}\left(O_{u}(r)\right)\right]\right\| d t \\
& \leq\left(2 C_{F}\|\Psi\|_{F} \int_{0}^{s} g(t) d t\right)\left\|O_{u}(r)\right\| \sum_{z \in \Lambda_{r^{\prime} \backslash \Lambda_{r^{\prime}-1}}} \sum_{x \in \Lambda_{0}} F_{\Psi}(d(x, z)) \\
& +2\left(2 C_{F}\|\Psi\|_{F} \int_{0}^{s} g(t) d t\right)\left\|O_{u}(r)\right\| \sum_{z \in \Lambda_{r^{\prime}-1} \backslash \Lambda_{\left\lfloor\left(r^{\prime}-1\right) / 3\right\rfloor}} \sum_{x \in \Lambda_{0}} F_{\Psi}(d(x, z)) \\
& +4 s\|\Psi\|_{F}\left\|O_{u}(r)\right\| \sum_{z \in \Lambda_{r^{\prime}-1} \backslash \Lambda_{\left\lfloor 2\left(r^{\prime}-1\right) / 3\right\rfloor}} \sum_{x \in \Lambda_{\left\lfloor\left(r^{\prime}-1\right) / 3\right\rfloor}} F_{\Psi}(d(x, z)) \\
& +16 s\|\partial \Phi\|_{a} F_{a}(0) \sum_{n \geq 1} \sqrt{G_{1}(n-1)}\left\|O_{u}(r)\right\|\left|\Lambda_{\left\lfloor 2\left(r^{\prime}-1\right) / 3\right\rfloor}\right| \sqrt{K\left(\left\lfloor\left(r^{\prime}-1\right) / 3\right\rfloor\right)},
\end{aligned}
$$

where the following functions are defined:

$$
F_{\Psi}(r)=\tilde{u}_{\mu}\left(\frac{\gamma^{\prime}}{8 v_{a}} r\right) F\left(\frac{\gamma^{\prime}}{8 v_{a}} r\right)
$$

with $F(r)=(1+r)^{-(d+1)}$ and,

$$
\begin{aligned}
& \tilde{u}_{\mu}(x)=\left\{\begin{array}{c}
u_{\mu}\left(e^{2}\right) \text { for } 0 \leq r \leq e^{2} \\
u_{\mu}(x)
\end{array}\right\} \\
& u_{\mu}(x)=\exp \left\{-\mu \frac{x}{\ln ^{2} x}\right\} \quad \text { for } \mu>0, \quad x>1 .
\end{aligned}
$$

Moreover,

$$
G_{1}(n) \leq 4 G_{2}\left(\frac{\gamma^{\prime} n}{2 v_{a}}\right)+\frac{C_{a}\|F\|}{a v_{a}} e^{-a n / 2}
$$

and

$$
K(x) \leq 4 G_{2}\left(\frac{\gamma^{\prime}|x|}{2 v_{a}}\right)+\frac{C_{a} C_{F_{a}}\|\Phi\|_{a}\|F\|}{a^{2} v_{a}^{2}} e^{-a x / 2}
$$


where $G_{2}(\zeta)$ is defined for $\zeta \geq 0$ by

$$
G_{2}(\zeta)=\frac{1}{\gamma^{\prime}} \cdot \begin{cases}7,354 & 0 \leq \zeta \leq 36,058 \\ 130 \mathrm{e}^{2} \zeta^{10} u_{2 / 7}(\zeta) & \zeta>36,058\end{cases}
$$

Finally, the remaining constants (e.g. $C_{F}=2^{d+1} \sum_{r \in \mathbb{Z}^{d}} F(r),\|\Psi\|_{F},\|\partial \Phi\|_{a}, F_{a}(0), a, v_{a}$, etc.) are defined in [3, Sec. 4], where one also sees that:

$$
2 C_{F}\|\Psi\|_{F} \int_{0}^{s} g(t) d t \leq e^{2 s\|\Psi\|_{F} C_{F}}-1 .
$$

We note that the decays involved (i.e. the functions $K(x)$ and $F_{\Psi}(r)$ ) are most likely not optimal, but we consider it an important exercise to give a rough estimate of the order of magnitude and the dependence to the spectral gap $\gamma^{\prime}$, appearing in Definition 4, of the constants involved in the decay of the perturbations we are studying. In any case, the relevant decay for our computation in Lemma 2 is dominated by tail estimates of the function $u_{\mu}\left(r^{\prime}\right)$, which defines a sub-exponential decay with $\mu \sim \gamma^{\prime}$. Here, we have sketched how one may derive rigorous estimates, using the tightest Lieb-Robinson bounds available at this point in time.

[1] D. Aharonov, I. Arad, Z. Landau, and U. Vazirani, Quantum Hamiltonian complexity and the detectability lemma. arXiv:1011.3445v5

[2] K. M. R. Audenaert, A sharp continuity estimate for the von Neumann entropy, J. Phys. A: Math. Theor. 40 8127 (2007). arXiv:quant-ph/0610146

[3] S. Bachmann, S. Michalakis, B. Nachtergaele, and R. Sims, Automorphic Equivalence within Gapped Phases of Quantum Lattice Systems. arXiv:1102.0842

[4] C. Borgs and R. Kotecký and D. Ueltschi, Low temperature phase diagrams for quantum perturbations of classical spin systems, Comm. Math. Phys. 181 (1996) 409-446.

[5] S. Bravyi and J. Haah, On the energy landscape of 3D spin Hamiltonians with topological order. arXiv:1105.4159

[6] S. Bravyi and M.B. Hastings, A short proof of stability of topological order under local perturbations. arXiv:1001.4363

[7] S. Bravyi, M.B. Hastings, and F. Verstraete, Lieb-Robinson Bounds and the Generation of Correlations and Topological Quantum Order, Phys. Rev. Lett. 97, 050401 (2006). arXiv:quant-ph/0603121

[8] S. Bravyi, M.B. Hastings, and S. Michalakis, Topological quantum order: stability under local perturbations, J. Math. Phys. 51, 093512 (2010). arXiv:1001.0344

[9] X. Chen, Z.-C. Gu, and X.-G. Wen, Classification of Gapped Symmetric Phases in $1 D$ Spin Systems, Phys. Rev. B 83, 035107 (2011). arXiv:1008.3745

[10] N. Datta, R. Fernández, and J. Fröhlich, Low-temperature phase diagrams of quantum lattice systems. I. Stability for quantum perturbations of classical systems with finitely-many ground states, J. Statist. Phys. 84, 455-534 (1996).

[11] N. Datta, R. Fernández, J. Fröhlich, and L. Rey-Bellet, Low-temperature phase diagrams of quantum lattice systems. II. Convergent perturbation expansions and stability in systems with infinite degeneracy, Helv. Phys. Acta 69, 752-820 (1996).

[12] M. Fannes, A continuity property of the entropy density for spin lattice systems, Comm. Math. Phys. 31 291-294 (1973).

[13] M. Fannes, B. Nachtergaele, and R. Werner, Finitely Correlated States on Quantum Spin Chains, Comm. Math. Phys. 144, 443-490 (1992).

[14] J. Haah, Local stabilizer codes in three dimensions without string logical operators, Phys. Rev. A 83, 042330 (2011). arXiv:1101.1962

[15] M.B. Hastings, Lieb-Schultz-Mattis in higher dimensions, Phys. Rev. B 69, 104431 (2004). arXiv:cond-mat/0305505

[16] M.B. Hastings, An area law for one dimensional quantum systems, JSTAT P08024 (2007). arXiv:0705.2024

[17] M.B. Hastings, Quasi-adiabatic Continuation for Disordered Systems: Applications to Correlations, Lieb-Schultz-Mattis, and Hall Conductance. arXiv:1001.5280

[18] M.B. Hastings Topological Order at Non-Zero Temperature arXiv:1106.6026

[19] M.B. Hastings and T. Koma, Spectral Gap and Exponential Decay of Correlations, Commun. Math. Phys. 265, no. 3, 781-804 (2006). arXiv:math-ph/0507008

[20] M.B. Hastings and S. Michalakis, Quantization of Hall conductance for interacting electrons without averaging assumptions. arXiv:0911.4706

[21] M. Hastings and X. Wen, Quasi-adiabatic continuation of quantum states: The stability of topological ground-state degeneracy and emergent gauge invariance, Phys. Rev. B 72, 045141 (2005). arXiv:cond-mat/0503554

[22] A.E. Ingham, A note on Fourier Transforms, J. London Math. Soc. 9, 29-32 (1934).

[23] T. Kennedy, H. Tasaki, Hidden symmetry breaking and the Haldane phase in $S=1$ quantum spin chains, Commun. Math. Phys. 147, 431-484 (1992).

[24] A. Kay, The capabilities of a perturbed toric code as a quantum memory. arXiv:1107.3940v2

[25] A. Kitaev, Fault-tolerant quantum computation by anyons, Ann. Phys. 303, 2-30 (2003). arXiv:quant-ph/9707021 
[26] I. Klich, On the stability of topological phases on a lattice, Ann. Phys. 325, 2120-2131 (2010). arXiv:0912.0945

[27] E.H. Lieb and D.W. Robinson, The finite group velocity of quantum spin systems, Comm. Math. Phys. 28, 251-257 (1972).

[28] B. Nachtergaele, The spectral gap for some quantum spin chains with discrete symmetry breaking, Commun. Math. Phys. 175, 565-606 (1996). arXiv:cond-mat/9410110

[29] B. Nachtergaele, Y. Ogata, and R. Sims. Propagation of Correlations in Quantum Lattice Systems, J. Stat. Phys. 124, 1-13 (2006). arXiv:math-ph/0603064

[30] B. Nachtergaele, H. Raz, B. Schlein, and R. Sims, Lieb-Robinson Bounds for Harmonic and Anharmonic Lattice Systems, Commun. Math. Phys. 286, 1073-1098 (2009). arXiv:0712.3820

[31] B. Nachtergaele and R. Sims, Lieb-Robinson Bounds and the Exponential Clustering Theorem, Commun. Math. Phys. 265, 119-130 (2006). arXiv:math-ph/0506030

[32] B. Nachtergaele and R. Sims, Locality Estimates for Quantum Spin Systems, in: V. Sidoravicius (Ed.), New Trends in Mathematical Physics. Selected contributions of the XVth International Congress on Mathematical Physics, Springer Verlag, 591-614 (2009). arXiv:0712.3318

[33] B. Nachtergaele and R. Sims, Lieb-Robinson Bounds in Quantum Many-Body Physics, in: R. Sims and D. Ueltschi (Eds), Entropy and the Quantum, Contemporary Mathematics, 529, 141-176 (2010). arXiv:0909.2845

[34] T.J. Osborne, Simulating adiabatic evolution of gapped spin systems, J. Phys. A. 75, 032321 (2007). arXiv:quant$\mathrm{ph} / 0601019$

[35] T.J. Osborne, private communication.

[36] D. Pérez-García, F. Verstraete, I. Cirac, and M. Wolf, PEPS as unique ground states of local Hamiltonians, Quant. Inf. Comp. 8, 0650-0663 (2008). arXiv:0707.2260

[37] I. Prémont-Schwarz, A. Hamma, I. Klich, and F. Markopoulou-Kalamara, Lieb-Robinson bounds for commutator-bounded operators, Phys. Rev. A. 81, 040102 (2010). arXiv:0912.4544

[38] I. Prémont-Schwarz and J. Hnybida, Lieb-Robinson bounds with dependence on interaction strengths, Phys. Rev. A. 81, 062107 (2010). arXiv:1002.4190

[39] S. Sachdev, Quantum phase transitions, Cambridge University Press, 2000.

[40] N. Schuch, I. Cirac, and D. Pérez-García, PEPS as ground states: Degeneracy and topology, Ann. Phys. 325, 2153-2192 (2010). arXiv:1001.3807

[41] N. Schuch, D. Pérez-García, and I Cirac, Classifying quantum phases using MPS and PEPS. arXiv:1010.3732

[42] W.L. Spitzer and S. Starr, Improved bounds on the spectral gap above frustration free ground states of quantum spin chains. Lett. Math. Phys., 63 165-177 (2003). arXiv:math-ph/0212029

[43] C. Stark, A. Imamoglu, and R. Renner, Localization of Toric Code Defects, Phys. Rev. Lett. 107, 030504 (2011). arXiv:1101.6028

[44] D. Yarotsky, Ground states in relatively bounded quantum perturbations of classical lattice systems, Commun. Math. Phys. 261, 799-819 (2006). arXiv:math-ph/0412040

[45] J. R. Wootton and J. K. Pachos, Bringing Order through Disorder: Localization of Errors in Topological Quantum Memories, Phys. Rev. Lett. 107, 030503 (2011). arXiv:1101.5900

[46] B. Yoshida, Feasibility of self-correcting quantum memory and thermal stability of topological order, Ann. Phys. In Press, Corrected Proof. arXiv:1103.1885 